University of Massachusetts Amherst

ScholarWorks@UMass Amherst

Masters Theses

Dissertations and Theses

November 2015

\title{
RNAi Validation of Resistance Genes and Their Interactions in the Highly DDT-Resistant 91-R Strain of Drosophila Melanogaster
}

Kyle Gellatly

University of Massachusetts Amherst

Follow this and additional works at: https://scholarworks.umass.edu/masters_theses_2

Part of the Biochemistry Commons, and the Molecular Biology Commons

\section{Recommended Citation}

Gellatly, Kyle, "RNAi Validation of Resistance Genes and Their Interactions in the Highly DDT-Resistant 91-R Strain of Drosophila Melanogaster" (2015). Masters Theses. 271.

https://doi.org/10.7275/7335178 https://scholarworks.umass.edu/masters_theses_2/271

This Open Access Thesis is brought to you for free and open access by the Dissertations and Theses at ScholarWorks@UMass Amherst. It has been accepted for inclusion in Masters Theses by an authorized administrator of ScholarWorks@UMass Amherst. For more information, please contact scholarworks@library.umass.edu. 


\title{
RNAI VALIDATION OF RESISTANCE GENES AND THEIR INTERACTIONS IN THE HIGHLY DDT-RESISTANT 91-R STRAIN OF DROSOPHILA MELANOGASTER
}

\author{
A Thesis Presented \\ By \\ KYLE GELLATLY
}

\begin{abstract}
Submitted to the Graduate School of the University of Massachusetts Amherst in partial fulfillment

of the requirements for the degree of
\end{abstract}

\section{MASTER OF SCIENCE}

September 2015

Molecular and Cellular Biology 


\title{
RNAI VALIDATION OF RESISTANCE GENES AND THEIR INTERACTIONS IN THE HIGHLY DDT-RESISTANT 91-R STRAIN OF DROSOPHILA MELANOGASTER
}

\author{
A Thesis Presented \\ By \\ KYLE GELLATLY
}

Approved as to style and content by:

John Clark, Chair

Jennifer Normanly, Member

Kathleen Arcaro, Member

Barbara Osborne, Director

Molecular and Cellular Biology Program 


\section{ACKNOWLEDGEMENTS}

I want to thank Dr. John Clark, who has facilitated my progression as an investigator over the last few years, and given me direction as a researcher.

I want to thank Dr. Jennifer Normanly for her consistent advising over the past 6 years of my college career and for being a committee member.

I want to thank Dr. Kathleen Arcaro for allowing me to use her lab resources and for her time and efforts for being a committee member.

I want to thank Dr. Jeff Doherty for introducing me to the MPAL community, and for all things related to chromatography.

I want to thank the rest of the Clark lab for discussing my project with me and being great friends.

I want to thank my family who has been so supportive over the last 6 years while I have been in Amherst.

I want to thank Nicole Moore who has sat through many discussions of what I was working on in lab, and always been supportive. 


\begin{abstract}
RNAI VALIDATION OF RESISTANCE GENES AND THEIR INTERACTIONS IN THE HIGHLY DDT-RESISTANT 91-R STRAIN OF DROSOPHILA MELANOGASTER
\end{abstract}

SEPTEMBER 2015

KYLE J. GELLATLY

\title{
B.S., UNIVERSITY OF MASSACHUSETTS AMHERST M.S., UNIVERSITY OF MASSACHUSETTS AMHERST
}

Directed by: Dr. John Marshall Clark

4,4'-dichlorodiphenyltrichloroethane (DDT) has been re-recommended by the World Health Organization for malaria mosquito control. Previous DDT use has resulted in resistance, and with continued use resistance will increase in terms of level and extent. Drosophila melanogaster is a model dipteran that has many available genetic tools, numerous studies done on insecticide resistance mechanisms, and is related to malaria mosquitoes allowing for extrapolation. The $91-R$ strain of $D$. melanogaster is highly resistant to DDT ( $>1500$-fold), however, there is no mechanistic scheme that accounts for this level of resistance. Recently, reduced penetration, increased detoxification, and direct excretion have been identified as resistance mechanisms in the $91-R$ strain. Their interactions, however, remain unclear. Use of UAS-RNAi transgenic lines of $D$. melanogaster allowed for the targeted knockdown of genes putatively involved in DDT resistance and has validated the role of several cuticular proteins (Cyp4g1 and Lcp1), cytochrome P450 monooxygenases (Cyp6g1 and Cyp12d1), and ATP binding cassette transporters (Mdr50, Mdr65, and Mrp1) involved in DDT resistance. 


\section{TABLE OF CONTENTS}

Page

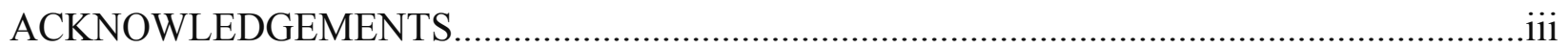

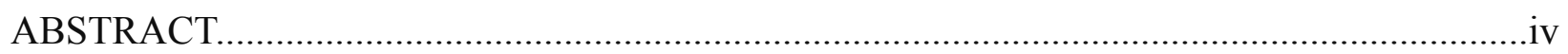

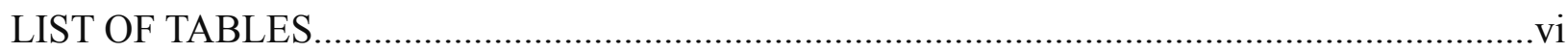

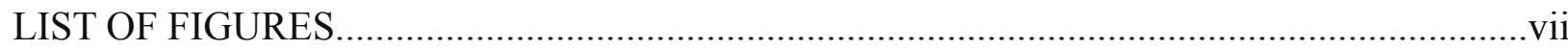

\section{CHAPTER}

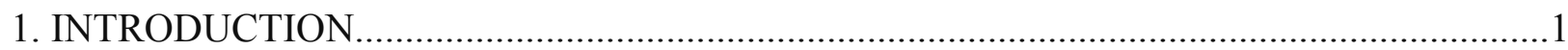

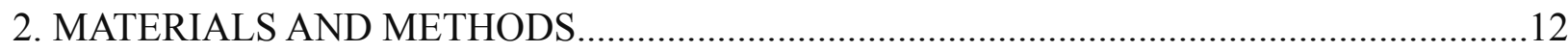

2.1 Drosophila melanogaster strains........................................................................12

2.2 Selection of genes for UAS-RNAi and dsRNA injection-induced RNAi................... 14

2.3 Total RNA extraction and reverse transcriptase-quantitative real-time PCR

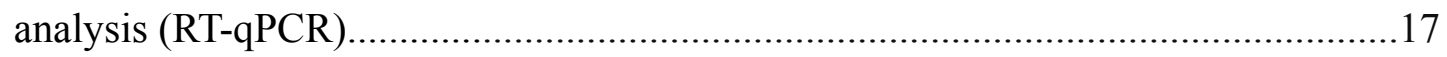

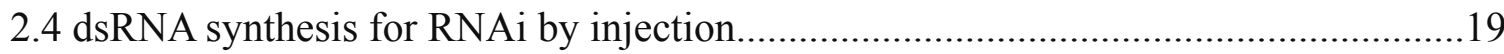

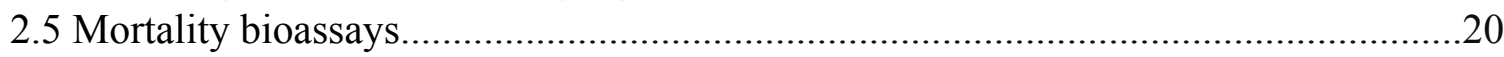

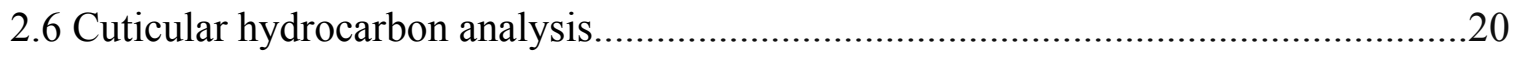

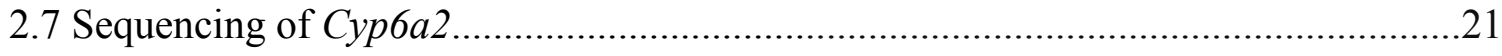

3. ROLE OF UAS-RNAi VALIDATED GENES IN DDT SENSITIVITY

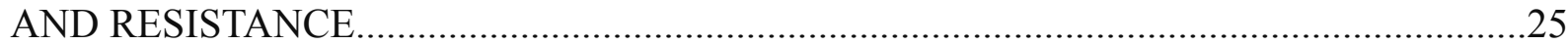

3.1 Role of cuticular genes in DDT sensitivity and resistance...................................25

3.2 Role of phase I or II metabolism genes in DDT sensitivity and resistance.................30

3.3 Role of phase III metabolism genes in DDT sensitivity and resistance.....................33

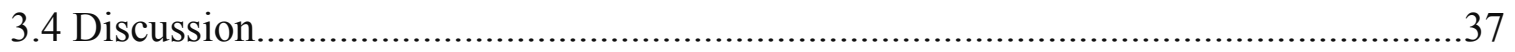

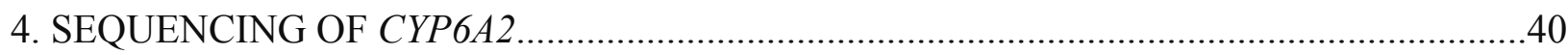

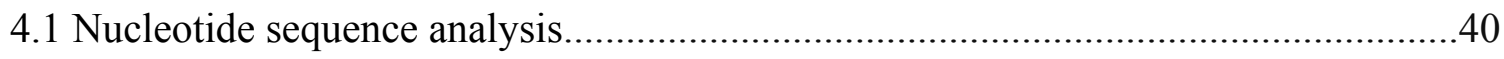

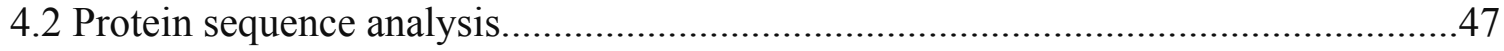

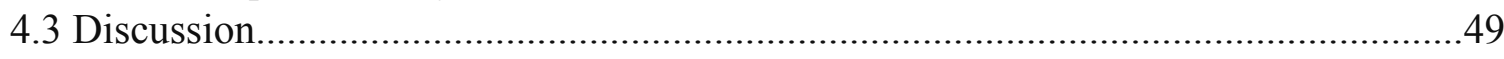

5. SUMMARY AND FUTURE DIRECTIONS ...................................................................50

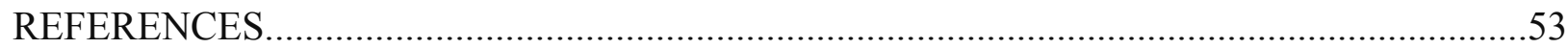




\section{LIST OF TABLES}

Table

Page

1. Fly lines used for UAS-RNAi genetic crosses.......................................................13

2. Genes selected for RNAi knockdown through literature search, toxicokinetic data, and bioinformatics approaches.

3. Search parameters used in Flybase for selection of candidate ABC transporter genes potentially involved in DDT efflux.

4. $\quad$ Primer sequences used for RT-qPCR experiments....................................................18

5. Sequence analysis of Cyp6a2 in DDT susceptible (CS, 91-C, UAS-Cyp6a2) and resistant (91-R, Cyp6a2SVL) strains led to the identification of several mutations in addition to SVL 


\section{LIST OF FIGURES}

Figure $\quad$ Page

1. The chemical structure of $4,4^{\prime}$-dichlorodiphenyltrichloroethane (DDT) .............................2

2. Through the cross of a Gal4 expressing driver and a transformant with an inverted repeat for a gene of interest, RNAi can be achieved...............................................................5

3. The protective barrier between the insect and its surrounding environment contains

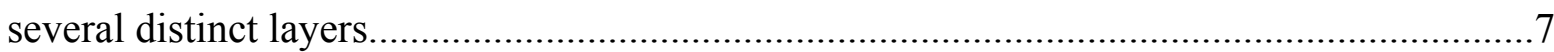

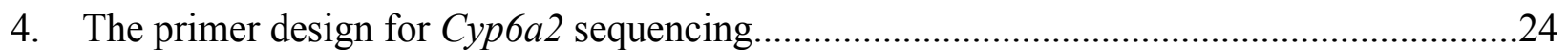

5. Cyp4g1 was over transcribed in the DDT-resistant $91-R$ strain compared to the insecticide-susceptible $C S$ strain.....

6. Cuticular hydrocarbon analyses by GC-FID of 3 biological replicates (15 females each) of crosses between Driver 1799 and UAS-RNAi line 102864 (Cyp4gl).

7. UAS-RNAi qPCR and mortality bioassay validation of genes putatively involved in the penetration of DDT.

8. UAS-RNAi qPCR and mortality bioassay validation of genes involved in the phase I or II xenobiotic metabolism of DDT.

9. UAS-RNAi qPCR and mortality bioassay validation of genes involved in the phase III metabolism of DDT.

10. The synthesis of gene specific dsRNAs for intra-abdominal injection induced RNAi against the ABC-Transporters Mdr49 (Lane 1, 553 bp), Mdr50 (Lane 2, 509 bp), Mdr65 (Lane 3, 508 bp), and Mrpl (Lane 4, 577 bp) consisted of two steps

11. Genes putatively involved in DDT resistance through literature and bioinformatics review validated through use of the UAS-RNAi system (number in parentheses are approximate chromosome locations in mega-basepairs as determined by NCBI Map Viewer).

12. Agarose gel showing the PCR product of Cyp6a2 for the CS, 91-C, UAS-Cyp6a2, and 91-R strains

13. The CLC DNA sequence alignments between the $C S, 91-C, 91-R$, UAS-Cyp6a2, and Cyp6a2SVL strains showed 21 SNP's were present in the coding region of Cyp6a2. 
14. The CLC DNA sequence alignments between the $C S, 91-C$, UAS-Cyp6a2, and 91- $R$ strains showed a $\sim 500 \mathrm{bp}$ insert in the 3 ' region of the gene that was not present in the DDT-resistant 91-R strain.

15. The CLC Protein sequence alignments between the $C S, 91-C, 91-R$, UAS-Cyp6a2, and Cyp6a2SVL strains showed 9 amino acid substitution in total 


\section{CHAPTER 1}

\section{INTRODUCTION}

4,4'-dichlorodiphenyltrichloroethane (DDT) (Fig. 1), a neurotoxic organochlorine insecticide that was phased out of use in the 1980s due to environmental impacts, has since been re-recommended by the World Health Organization for use indoors to control mosquito populations in malaria prone areas $[1,2]$. Malaria, an infectious disease spread through mosquito vectors, was responsible for nearly 655,000 deaths in 2010 , the majority being less than 5 years of age [3]. Through both indoor residual spraying (IRS) and insecticide-treated nets (ITNs), DDT has been shown to be effective at reducing malaria transmission rates in disease prone areas. Its relatively low cost, long term effectiveness, and lack of environmental impact when applied properly, has led to fourteen African Sub-Saharan countries, and others around the globe such as India, to once again use DDT as a preventative measure against malaria.

This widespread use, however, has come at a cost; by 1984, a world survey showed that 233 species, mostly insects, were resistant to DDT [4]. Even more alarming is that DDT resistance has been reported in more than 50 species of anopheles mosquitoes, many of which are vectors of malaria [2]. The use of DDT for vector control continues to this day, and its use will increase as insect-borne diseases expand [5,6]. Drosophila melanogaster (D. melanogaster), a genetic model organism for the last several decades with many genetic tools and a wealth of information about insecticide resistance mechanisms [7], has also been shown to be highly resistant to DDT [8-11]. Both the mosquito and D. melanogaster belong to order Diptera, and because they are highly related genetically, studies done on D. melanogaster can be extrapolated to the mosquito as well [12]. 


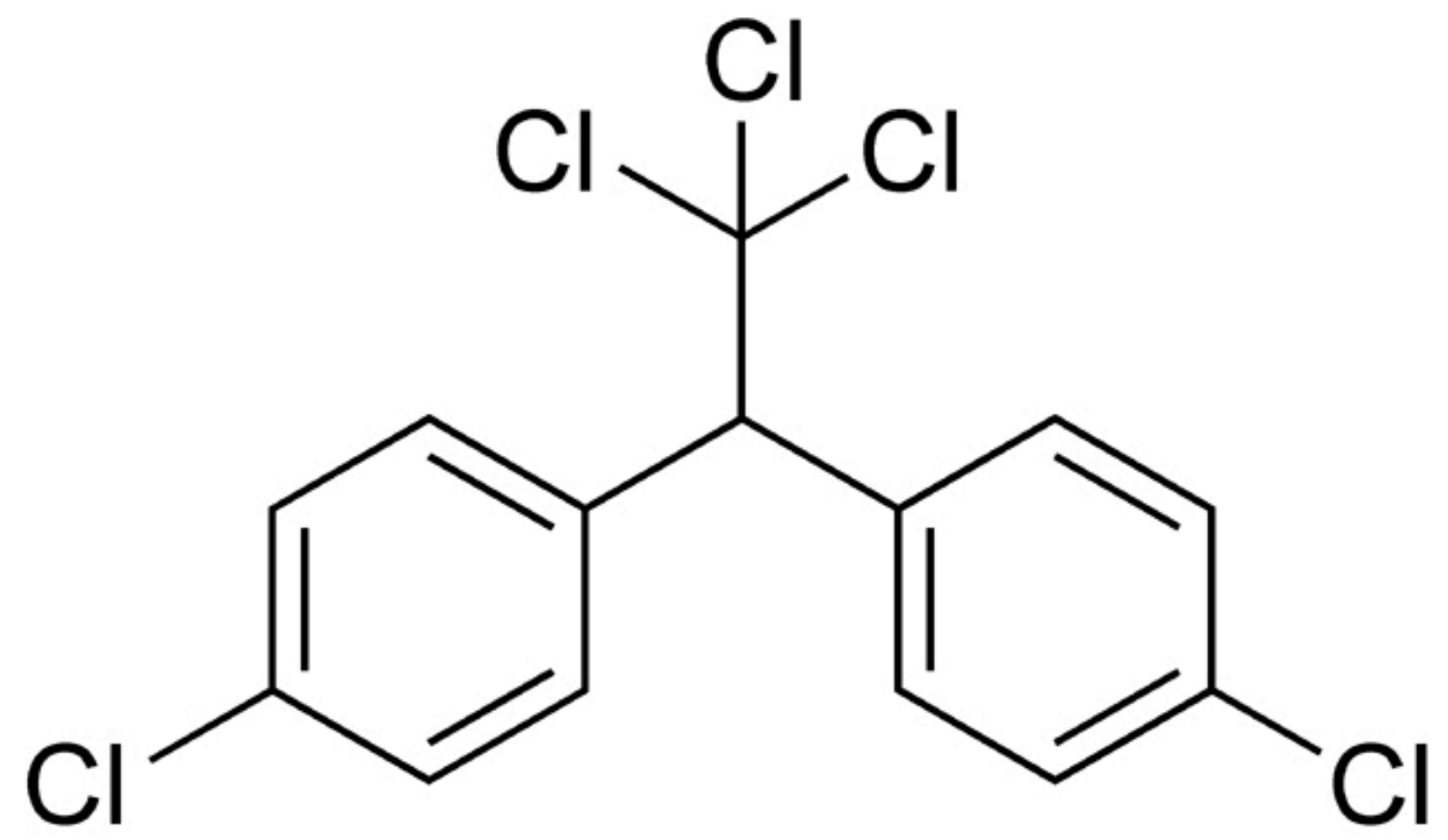

Figure 1. The chemical structure of 4,4'-dichlorodiphenyltrichloroethane (DDT). 
Several strains of $D$. melanogaster have often been used in DDT resistance studies, such as the Canton-S (CS, DDT-susceptible), 91-C (slightly DDT-resistant), and the 91-R strain (highly DDT-resistant). The highly DDT-resistant $91-R$ strain of $D$. melanogaster is over 1500fold more resistant to DDT than CS [13]. As of yet, however, there is no definitive mechanistic model to accurately account for this level of resistance. Genome-wide transcription profiling, as well as protein mapping, has suggested that there are a number of factors involved in DDT resistance [14-16]. Both target-site insensitivity and enhanced xenobiotic metabolism have been shown in some cases to be responsible for DDT resistance [17-19]. It has been previously reported that the increased cellular excretion of DDT in the $91-R$ strain is caused by increased expression of ATP-Binding Cassette Transporters (ABC-transporters) and the increased metabolism of DDT is caused in part by over expression of cytochrome P450 monooxygenase (P450s) [13]. With mortality bioassays, it was estimated that increased excretion and metabolism conferred 10- and 2.2-fold resistance levels, respectively. In the same investigation, a penetration factor was attributed to a 68 -fold increase in resistance. Using gas-liquid chromatography with flame ionization detection (GC-FID), 5 cuticular hydrocarbons were identified to be significantly more abundant $(\mathrm{p}<0.05)$ in the $91-R$ strain compared with $C S$ flies. It is likely that the penetration factor associated with DDT resistance is due, in part, to the increase in cuticular hydrocarbons in the epicuticle of $91-R$ flies. However, the molecular mechanism of this resistance factor had not yet been identified.

In 2007, a large step forward was taken towards the ultimate goal of identifying the role of nearly every gene in D. melanogaster by creating a transgenic RNAi library that contained transformants for $97 \%$ of all genes in D. melanogaster [20]. The RNAi library was created by 
inserting gene activation control elements that are normally found in yeast into the $D$. melanogaster genome. This yeast system, called Ga14/UAS, works through the combination of two distinct parts: Gal4 and the upstream activation sequence (UAS). Gal4 is a modular protein in yeast that is responsible for DNA binding in order to activate gene expression. The UAS is a sequence before the coding region of the gene to which Gal4 binds to initiate transcription (Fig. 2) $[21,22]$.

In D. melanogaster, lines were generated that expressed Gal4 in a variety of different ways: including constitutive, time specific, tissue specific, or inducible through chemical or environmental conditions such as a heat shock. Transformants were also created that contained an UAS preceding gene specific inverted repeats. When induced, these insertions express RNA that folds back onto itself to form a dsRNA hairpin loop, which is processed by RNAi machinery to lead to gene silencing [23]. By crossing a driver strain (which contains a Gal4 expression insertion) with a transformant (that contains an inverted repeat of a target gene under Gal4 promoter), RNAi can be induced in the resulting F1 progeny. 
Driver: GAL4 expressing line
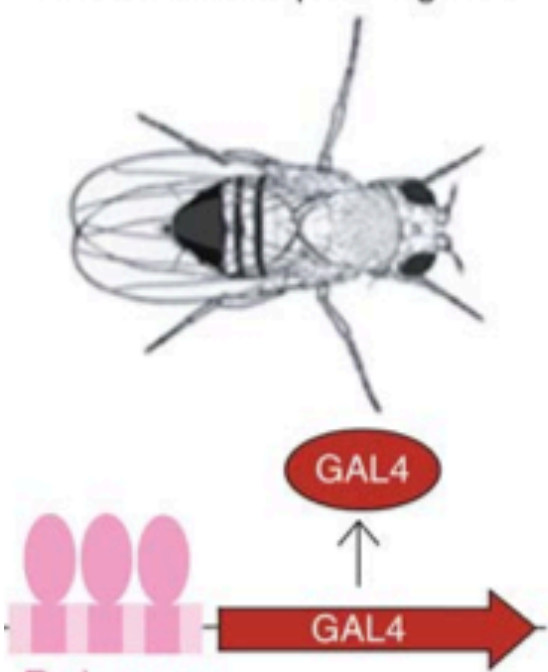

Endogenous promoter/ enhancer

GAL4 expressed in cell- or tissuespecific pattern
Responder: UAS-target gene line

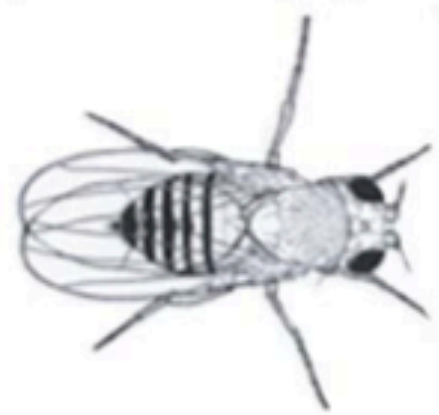

$\mathrm{X}$

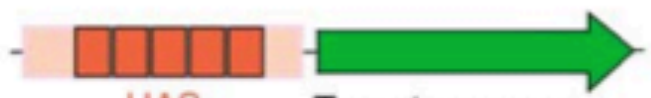

UAS

(GAL4 binding

Target sequence

sites)

UAS-target gene silent in absence of GAL4

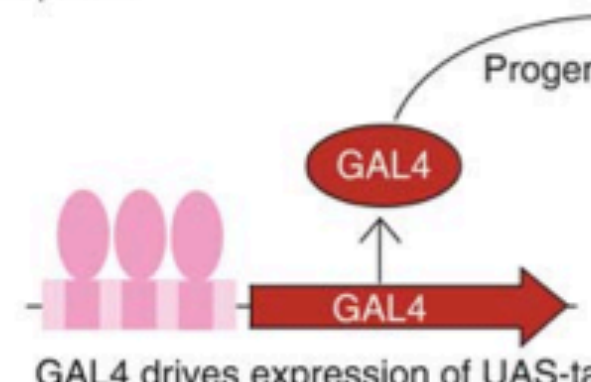

GAL4 drives expression of UAS-target gene in cell- or tissue-specific pattern

Figure 2. Through the cross of a Gal4 expressing driver and a transformant with an inverted repeat for a gene of interest, RNAi can be achieved. The expression of the dsRNA is processed by RNAi machinery to lead to gene silencing. [22] 
Decreased penetration, increased xenobiotic metabolism, and increased xenobiotic excretion have all been shown to be at least partially responsible for overall DDT resistance levels in the $91-R$ strain of D. melanogaster [13]. Before xenobiotic metabolism can take place, however, DDT must first penetrate into the target organism [24]. Decreased cuticular penetration therefore can impede the rate of distribution of DDT into the hemolymph [25] and ultimately the nervous system of the insect. In order for this to occur, DDT must pass through a variety of different layers of the insect cuticle that make up the protective barrier between the fly and its surrounding environment (Fig. 3). First, there is an epicuticle, which contains among other components, an external layer of waxy cuticular hydrocarbons that have evolved to prevent desiccation [26]. This external barrier is followed by the exocuticle and endocuticle, which consists of various cross-linked proteins and chitin. Lastly, there is a layer of epidermal cells that are responsible for the production and excretion of many of the proteins and compounds that are found within the cuticle itself [27]. A specialized cell type within the epidermis, the oenocyte, is responsible for the decarbonylation of long chain aldehydes, which are then transported to the outer surface by way of pore canals to form the waxy cuticular hydrocarbon layer [28].

Decreased penetration is an important factor for DDT resistance. Due to its lipophilicity, the ability of DDT to penetrate through the cuticular waxy hydrocarbon layer is largely dependent on the amount of hydrocarbon present. Changes in the protein content of the cuticle itself may also be responsible for the decreased rate of penetration, such as changes in the chitin content [29], laccase gene expression [30], or larval cuticle proteins such as Lcp1 [31]. There is a strong correlation between the presence of this chitinous cuticle and sensitivity to DDT [32]. It has been hypothesized that an increase in cuticular hydrocarbons would decrease the rate by which DDT can penetrate into the target organism giving rise to resistance [13]. 


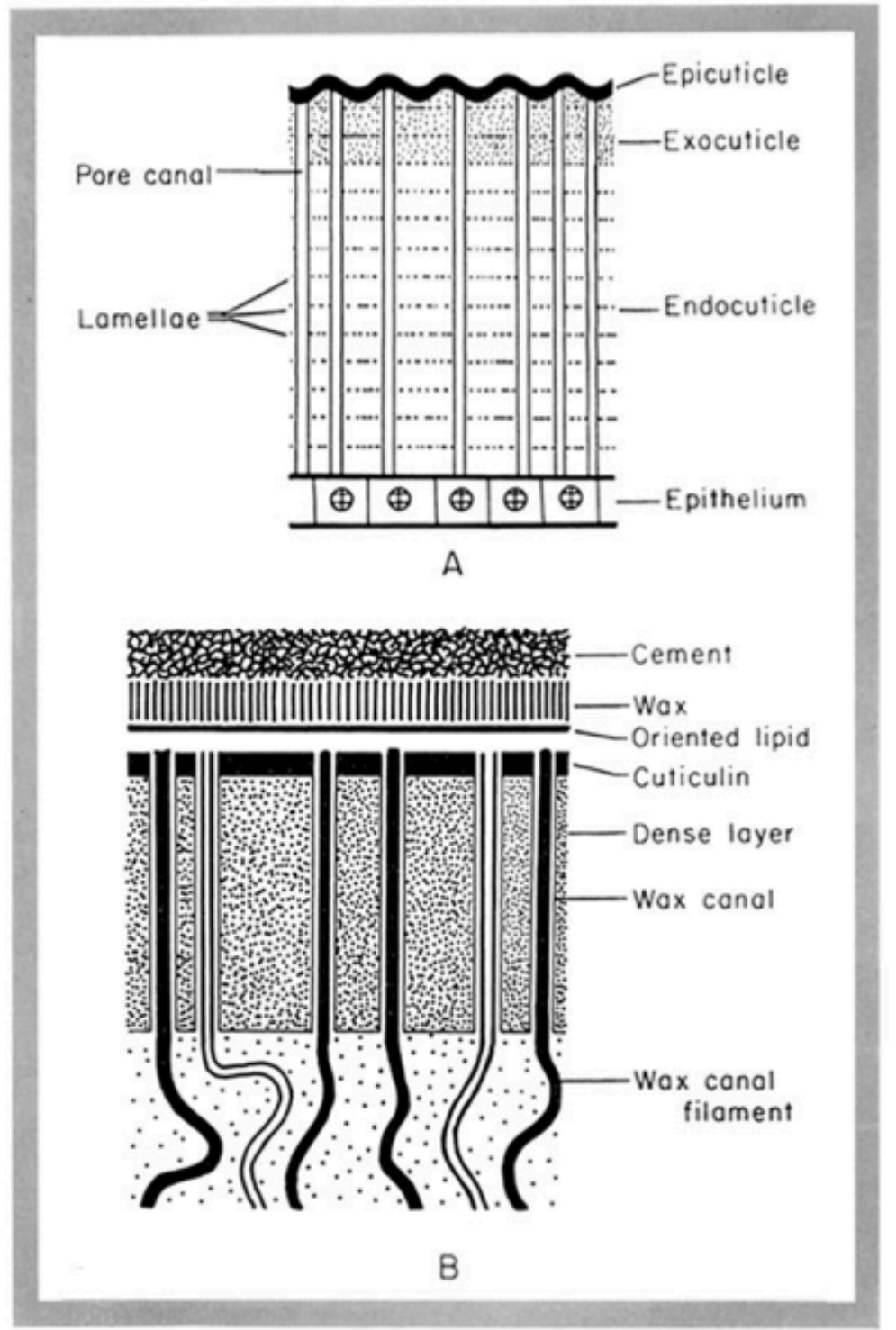

Figure 3. The protective barrier between the insect and its surrounding environment contains several distinct layers. Panel A shows the entire insect cuticle, while panel B is an inset of the epicuticle. The typical insect cuticle is composed of an outer epicuticle, which contains the waxy cuticular hydrocarbons to prevent desiccation, followed by the exo and endocuticle. These layers are produced by an underlying layer of epidermal cells. The oenocyte, a specialized epidermal cell, is responsible for the production of cuticular hydrocarbons. [33] 
Reductive metabolism of DDT has been shown to occur in several P450 systems [34,35]. Increased metabolism, as a mechanism of DDT resistance in D. melanogaster, has been shown to be largely due to either increased expression or structural changes in P450s [14]. Several isoforms of P450s metabolize DDT into its primary and secondary metabolites. Specifically, Cyp6g1, Cyp12d1, Cyp6a8, and Cyp6a2 have been shown to be significantly over expressed in several DDT-resistant strains [15, 36-39]. Microarray analysis showed that Cyp6gl was inducible by DDT [40]. DDT has also been shown to be metabolized by Cyp6gl expression in cell cultures of Nicotiana tabacum [41]. Further, when Cyp6g1 expression was induced in D. melanogaster using a Gal4/UAS system, a greater level of DDT resistance was demonstrated $[42,43]$. Investigations into the reason behind the over expression of Cyp $6 g 1$ have led to the identification of cis-acting elements [44]. Some suggest that it is due to the Accord retrotransposon, which results in tissue specific over expression [45-47]. While Cyp6g1 may be considered by some to be the only P450 responsible for DDT metabolism in D. melanogaster, there are others who argue against this suggestion. One study found that Cyp6gl over expression alone does not confer DDT resistance in D. melanogaster [48], while another study reported that Cyp6gl knockout has no effect on DDT susceptibility [49].

While Cyp6g1 appears to be important in DDT metabolism, there are several other P450 candidates that may lead to the extremely high level of resistance found in $91-R$ flies. Cyp $12 d 1$ is one such candidate. Several studies have shown that Cyp $12 d 1$ is over expressed in resistant lines, and is inducible by DDT exposure [37,50]. Transgenic over expression of Cyp12d1 resulted in increased survival times of flies exposed to DDT [43]. Other P450s of interested include Cyp6a8 and Cyp6a2. Cyp6a8 has been shown to be over expressed in DDT-resistant flies, likely due to 
factors in the 5' region of the gene [51]. Both Cyp6a8 and Cyp6a2 are constitutively over expressed, as well as being inducible with phenobarbital $[52,53]$. Another study suggests that the over expression of these genes is actually due to factors found on the third chromosome [38]. Cyp6a2 has had several studies done on its ability to metabolize DDT. One study shows that while it is over expressed in resistant flies, its ability to be induced by insecticides is actually greater in susceptible flies [54]. Some studies looking to identify the reason for Cyp6a2 over expression have suggested that it is due to either 5' transcriptional binding sites or changes in the 3 ' region of the gene $[51,55]$.

The ability to metabolize DDT using wild-type Cyp6a2 seems to be negligible, while a R335S, L336V, V476L, (Cyp6a2SVL) mutant form has been reported to metabolize DDT when transformed into Escherichi coli [56]. Expression of the Cyp6a2SVL mutant was shown to increase the production of DDA, DDD, and dicofol, known DDT metabolites. The highly DDTresistant $91-R$ strain was shown to produce greater amounts of dicofol and DDD, as well as 2 unidentified metabolites (perhaps one of which may be DDA), compared to the DDT-susceptible CS. As Cyp6a2 is the most highly over expressed P450 in the $91-R$ strain, it would make sense to offset that energetic cost with the fitness advantage of direct DDT metabolism and detoxification. However, because wild type Cyp6a2 does not metabolize DDT, there is a possibility that the 91 $R$ strain of D. melanogaster contains the SVL mutations. Sequencing Cyp6a2 across the CS, 91C, 91-R, UAS-Cyp6a2 (Cyp6a2 RNAi line) strains and comparing them to the Cyp6a2SVL strain would show if these mutations are present in the DDT-resistant $91-R$ strain. The possibility remains, nevertheless, that additional Cyp6a2 mutations may exist in the $91-R$ strain which may enable DDT metabolism even if the SVL mutations are not present. 
With increased excretion driven by phase III metabolism, xenobiotics are effluxed out of the cell by ABC transporters, a class of proteins that span membranes and use ATP to drive this efflux [57]. ABC transporters have been shown to be an insecticide resistance mechanism in several insects [58-61]. Several ABC transporters have been implicated in DDT resistance in the 91-R flies such as Mdr49, Mdr50, Mdr65, and Mrp1 [62-65]. The multiple drug resistance (Mdr) genes are $\mathrm{ABC}-\mathrm{B}$ type transporters, while the multidrug resistance-associated proteins $(\mathrm{Mrp})$ are ABC-C type transporters. Over transcription of p-glycoproteins ( $M d r$, ABC-B type transporters) in epidermal cells has previously been shown to lead to insecticide resistance [66]. The first identification and characterization of D. melanogaster ABC-B transporters homologues occurred in 1991 and reported that they were similar to the mammalian $M d r$ cell lines [65]. Mdr49 was shown to be expressed in all stages of the flies' life cycle, suggesting that it may be one of the transporters involved in the efflux of DDT [67]. Similarly, a study that compared the expression of Mdr49 and Mdr65 showed that they were inducible by stress, including pesticides that are well known inducers of stress [68]. The identification of another $M d r$ gene, $M d r 50$ [64] showed that $D$. melanogaster had a variety of different $\mathrm{ABC}$ transporters in its genome, any of which may be responsible for effluxing DDT. Mrpl was identified as an orthologue of human MRP1, MRP2, MRP3, and MRP6, which were known to efflux a variety of therapeutic agents [69]. Further, Mrpl was shown to be inducible by DDT exposure [70].

Once DDT has penetrated the insect exoskeleton and is distributed within the hemolymph, both metabolism and excretion work cooperatively in an additive manner [71]. Penetration, however, has been described as a multiplicative resistance mechanism. Resistance to DDT in $91-R$ flies is extremely high and is likely due to a combination of several of the 
aforementioned mechanisms. Contact penetration of DDT was $\sim 30 \%$ less with $91-R$ flies, possibly due to having significantly more cuticular hydrocarbons and a thicker, more laminated cuticle compared to $C S$ flies [13]. DDT was metabolized $\sim 1$-6-fold more extensively by $91-R$ than $C S$ flies, resulting in dicofol, dichlorodiphenyldichloroethane (DDD), two unidentified metabolites and a number of unresolved polar conjugates being formed in significantly greater amounts [13]. 91-R flies also excreted $\sim 4$-fold more DDT and its metabolites than $C S$ flies [13]. Verapamil pretreatment reduced the LD50 value for $91-R$ flies topically dosed with DDT by a factor of 10-fold, indicating that the increased excretion may involve ABC B-type transporters [13]. Thus, DDT resistance in 91- $R$ flies is multifactorial and includes mechanisms involved in reduced penetration, increased detoxification and direct excretion, some of which may interact synergistically and result in high levels of DDT resistance.

Through crosses between a driver strain that expressed Gal4 under a heat shock promoter and UAS-RNAi lines for genes that were putatively determined to be involved in DDT resistance in the 91- $R$ strain, we identified several cuticular proteins, $\mathrm{P} 450 \mathrm{~s}$ and $\mathrm{ABC}$ transporters involved in DDT resistance across all three chromosomes. Our working hypothesis is that RNAi knockdown of the selected genes in the insecticide-susceptible transgenic flies, which results in increased sensitivity to DDT, are likely to function as resistance factors in 91- $R$ flies where these genes are over transcribed. 


\section{CHAPTER 2}

\section{MATERIALS AND METHODS}

\subsection{Drosophila melanogaster strains}

The DDT-resistant $91-R$ and insecticide-susceptible $C S$ strains were obtained from Dr. Barry Pittendrigh (University of Illinois, Urbana-Champaign). The 91-R strain has been shown to be $\sim 1500$-fold more resistant to DDT than susceptible strains through the use of contact bioassays [13]. The $91-R$ strain has been continually selected by maintaining the flies in a colony jar in the presence of a $150 \mathrm{mg}$ DDT/filter paper disk. All strains were reared at room temperature on Jazz-Mix Drosophila Food $^{\circledR}$ from Fisher Scientific (Waltham, MA) prepared according to the labeled instructions. Flies were kept in colony vials, which were changed every 2 weeks. Flies were anesthetized for transfers by placing dry ice in a Büchner flask with attached tubing connected to a needle that released $\mathrm{CO}_{2}$.

For heat shock experiments, virgin females of Driver 1799 from the Bloomington Drosophila Stock Center (BDSC) at Indiana University, Bloomington, IN, were selected. This driver strain contains a Gal4 insertion under the Hsp70 promoter, allowing Gal4 expression when heat shocked. Driver 1799 females were crossed with males of the UAS-RNAi lines from the BDSC and the Vienna Drosophila RNAi Center (VDRC, Vienna, Austria) (Table 1). These lines contain an inverted gene repeat under control of the Gal4 promoter region, which when expressed results in the formation of dsRNA hairpin loop structures [20,21]. Thus, a $1799 \mathrm{X}$ UAS-RNAi cross results in the generation of dsRNA for RNAi only after heat shock and allows for control groups (non-heat shocked, NHS) to be of the same genetic background as the RNAi groups (heat shocked, HS). 


\section{Table 1}

Fly lines used for UAS-RNAi genetic crosses.

\begin{tabular}{cccccc}
\hline Gene Class & CG\# & Synonym & Stock \# & Line Type & Vendor \\
\hline N/A & N/A & N/A & 1799 & Gal 4 Driver & BDSC \\
Cuticular & 11650 & Lcpl & 106030 & UAS-RNAi & VDRC \\
Protein & 4784 & Cpr72Ec & 29452 & UAS-RNAi & VDRC \\
P450 & 3972 & Cyp4g1 & 102864 & UAS-RNAi & VDRC \\
& 8453 & Cyp6gl & 104171 & UAS-RNAi & VDRC \\
& 30489 & Cyp12d1 & 109256 & UAS-RNAi & VDRC \\
& 10248 & Cyp6a8 & 100459 & UAS-RNAi & VDRC \\
& 8859 & Cyp6g2 & 105333 & UAS-RNAi & VDRC \\
& 9438 & Cyp6a 2 & 108776 & UAS-RNAi & VDRC \\
ABC B-type & 3879 & Mdr49 & 108327 & UAS-RNAi & VDRC \\
& 8523 & Mdr50 & 51166 & UAS-RNAi & VDRC \\
& 10181 & Mdr65 & 35035 & UAS-RNAi & BDSC \\
& 11897 & N/A & 105174 & UAS-RNAi & VDRC \\
& 7806 & N/A & 2804 & UAS-RNAi & VDRC \\
& 5789 & N/A & 1204 & UAS-RNAi & VDRC \\
ABC C-type & 6214 & Mrp1 & 105419 & UAS-RNAi & VDRC \\
Glutathione $S$ - & 17530 & GstE5 & 100632 & UAS-RNAi & VDRC \\
Transferase & 17527 & GstE6 & 25270 & UAS-RNAi & VDRC \\
\hline
\end{tabular}

For 1799 X UAS-RNAi line crosses, fly rearing vials were emptied of all adult flies and newly eclosed virgin females were selected within $6 \mathrm{~h}$ of adult emergence. Mating pairs of virgin females and males were transferred to new vials, and the mating pairs removed after $24 \mathrm{~h}$. This process ensured that the resulting F1 progeny were within $24 \mathrm{~h}$ of each other in age. All references to age were based on the number of days since egg oviposition. For all heat shock experiments, 9 day old flies were placed into a $37^{\circ} \mathrm{C}$ incubator for $45 \mathrm{~min}$ and then allowed to continuously develop under the standard rearing conditions described above. 


\subsection{Selection of genes for UAS-RNAi and dsRNA injection-induced RNAi}

Genes selected for RNAi knockdown (Table 2) were determined through a combination of literature searches, toxicokinetic analysis [13] and bioinformatic approaches (Table 3).

Table 2

Genes selected for RNAi knockdown through literature search, toxicokinetic data, and bioinformatics approaches.

\begin{tabular}{cccccc}
\hline Gene Class & CG\# & Synonym & Transcript Ratio & P - value & Selection Criteria \\
\hline Cuticular & 11650 & Lcp1 & 2.52 & .0067 & {$[38]$} \\
Protein & 4784 & Cpr72Ec & 4.39 & $<0.0001$ & {$[38]$} \\
Cytochrome & 3972 & Cyp4g1 & $*$ & $*$ & {$[25]$} \\
P450 & 8453 & Cyp6g1 & 2.83 & 0.0136 & {$[10,16,17,20,38]$} \\
& 30489 & Cyp12d1 & 0.1 & $<0.0001$ & {$[15,17,38]$} \\
& 10248 & Cyp6a8 & $*$ & $*$ & {$[14,15,50,51]$} \\
& 8859 & Cyp6g2 & 1.66 & .205 & {$[38,51]$} \\
& 9438 & Cyp6a2 & 34.3 & $<0.0001$ & {$[12,38,49,50,51]$} \\
ABC-B Type & 3879 & Mdr49 & $*$ & $*$ & {$[39,40]$} \\
& 8523 & Mdr50 & $*$ & $*$ & {$[39,41]$} \\
& 10181 & Mdr65 & $*$ & $*$ & {$[39,42]$} \\
& 11897 & N/A & $*$ & $*$ & Bioinformatics \\
& 7806 & N/A & $*$ & $*$ & Bioinformatics \\
& 5789 & N/A & $*$ & $*$ & Bioinformatics \\
ABC-C Type & 6214 & Mrp1 & $*$ & $*$ & {$[39,43]$} \\
Glutathione S- & 17530 & GstE5 & 3.03 & $<0.0001$ & {$[38]$} \\
Transferase & 17527 & GstE6 & 2.52 & 0.0016 & {$[38]$} \\
\hline
\end{tabular}

* No information on 91-R transcript levels.

Lcp1, Cpr72Ec, Cyp6g1, Cyp12d1, Cyp6a2, GstE6, and GstE5 have all been referenced in the literature as over transcribed in DDT-resistant strains [15]. Lcpl and Cpr72Ec are cuticular protein genes and increased expression of these genes in DDT-resistant strains was suggested to confer DDT resistance by altering the physical structure of the cuticle, making it more difficult for DDT to penetrate [13]. Cyp4gl was selected due to its recent identification as the P450 in oenocytes of the epidermis responsible for the decarbonylation of long chain aldehydes to form 
cuticular hydrocarbons [28]. Because 91- $R$ flies have an increased amount of cuticular hydrocarbons, Cyp4gl was a logical choice as being responsible for the increase in hydrocarbon abundance [13]. Cyp6g1, Cyp12d1, and Cyp6a2 were also shown to be over transcribed P450 genes in DDT-resistant strains, and these P450s could confer DDT resistance through direct metabolism and detoxification of DDT to dicofol or DDD [13]. Further, Cyp6a2 has been reportedly able to metabolize DDT [56], although this was only in a Cyp6a2SVL mutant form of the gene. Cyp6a2, Cyp6g2, and Cyp6a8 were also shown to be over expressed due to changes in the promoter and or 3' region of the genes [39]. The glutathione-S-transferase genes, GstE5 and GstE6, were found to be over expressed in the $91-R$ strain and are likely necessary to offset the increased oxidative stress produced through constitutive over transcription of the P450 detoxification genes [15].

The ABC B-type multiple drug resistance (Mdr) genes, $M d r 49, M d r 50, M d r 65$ as well as the ABC C-type multidrug resistance-associated protein Mrpl, were selected for RNAi due to preliminary reverse transcription-quantitative real time PCR (RT-qPCR) results that showed their over transcription in the $91-R$ strain [62] and their prior implications in insecticide phase III xenobiotic metabolism (efflux) and resistance [63-69]. Additional ABC B-type transporter genes, CG11897, CG7806, and CG5789, were selected through a bioinformatic approach using Flybase. Search parameters, such as xenobiotic transporting ATPase activity and expression in the Malpighian tubules (Table 3), were tabulated and the overall match scores determined by comparison with the known DDT interacting genes such as Mdr50. Any genes which had 4 or more positive parameters were deemed worthy of investigation. 
Table 3

Search parameters used in Flybase for selection of candidate ABC transporter genes potentially involved in DDT efflux.

\begin{tabular}{|c|c|c|c|c|c|c|c|c|c|}
\hline Family & Gene & Alias & $\begin{array}{c}\text { Xenobiotic } \\
\text { transporting ATPase } \\
\text { activity }\end{array}$ & $\begin{array}{l}\text { Drug transmembrane } \\
\text { transporter activity }\end{array}$ & Resistance & Drug & $\begin{array}{l}\text { Malphigian } \\
\text { tubules }\end{array}$ & Fat body & \# Positive Parameters \\
\hline A & (10) Genes & - & No & No & No & No & - & - & 0 \\
\hline B & CG10181 & Mdr65 & Yes & Yes & Yes & Yes & Yes & & 5 \\
\hline B & CG10226 & & & Yes & & & Yes & & 2 \\
\hline B & CG10441 & & & & & & & & \\
\hline B & CG17338 & & Yes & & & & & & \\
\hline B & CG1824 & & & & & & & & \\
\hline B & CG3156 & & Yes & Yes & & & & & 2 \\
\hline B & CG3879 & $M d r 49$ & & Yes & Yes & Yes & Yes & Yes & 5 \\
\hline B & CG4225 & & & & & & & & \\
\hline B & CG7955 & & & & & & & & \\
\hline B & CG8523 & Mdr50 & & Yes & Yes & Yes & & & 3 \\
\hline $\mathrm{C}$ & CG10505 & & & & & & & & \\
\hline $\mathrm{C}$ & CG11897 & & Yes & Yes & & Yes & Yes & Yes & 5 \\
\hline $\mathrm{C}$ & CG11898 & & Yes & Yes & & Yes & & & 3 \\
\hline $\mathrm{C}$ & CG14709 & & & & & & & & \\
\hline $\mathrm{C}$ & CG4562 & & & & & & & & \\
\hline $\mathrm{C}$ & CG5772 & & & & & & & & \\
\hline $\mathrm{C}$ & CG5789 & & Yes & Yes & & & Yes & Yes & 4 \\
\hline $\mathrm{C}$ & CG6214 & Mrpl & Yes & Yes & Yes & & Yes & Yes & 5 \\
\hline $\mathrm{C}$ & CG7627 & & Yes & Yes & & & & Yes & 3 \\
\hline $\mathrm{C}$ & CG7806 & & Yes & Yes & & & Yes & Yes & 4 \\
\hline $\mathrm{C}$ & CG8799 & & & & & & & & \\
\hline $\mathrm{C}$ & CG9270 & & Yes & Yes & & & Yes & & 3 \\
\hline D & (2) Genes & - & No & No & No & No & - & - & 0 \\
\hline E & (4) Genes & - & No & No & No & No & - & - & 0 \\
\hline G & (15) Genes & - & No & No & No & No & . & - & 0 \\
\hline $\mathrm{H}$ & $\begin{array}{l}\text { (3) Genes } \\
56 \text { Total }\end{array}$ & - & No & No & No & No & - & - & 0 \\
\hline
\end{tabular}




\subsection{Total RNA extraction and reverse transcriptase-quantitative real-time PCR analysis (RT-qPCR)}

For all analyses, 30 females (14 days post oviposition) were collected per biological replicate and 3 biological replicates were analyzed. This time point ensured that all heat shocktreated flies had fully emerged from their pupae. Flies were placed into $2 \mathrm{ml}$ vials with five 0.25 g stainless steel beads, loaded into an aluminum casing and homogenized with the GenoGrinder 2010 (SPEX Sample Prep, Metuchen, NJ) for $1 \mathrm{~min}$ at 1000 strokes per min. After homogenization, the total RNA was extracted using the Qiagen RNeasy kit (Qiagen, Valencia, CA) according to the manufacturer's instructions with an on column DNase digestion. Total RNA was eluted from the provided column in RNAse free (DEPC-treated), double-distilled $\mathrm{H}_{2} \mathrm{O}$ $\left(\mathrm{ddH}_{2} \mathrm{O}\right)$. The concentration of RNA was determined using the NanoDrop 8000 (NanoDrop Technologies, Wilmington, DE), and then adjusted to a final concentration of $100 \mathrm{ng} \mathrm{RNA} / \mu \mathrm{L}$.

$500 \mathrm{ng}$ of total RNA served as template for the first strand cDNA synthesis reaction using the iScript cDNA kit from Bio-Rad (Hercules, CA) in a $20 \mu \mathrm{L}$ reaction volume following the manufacturer's instructions. The resulting cDNA was used as a template in the RT-qPCR reaction. RT-qPCR analyses were performed using the Power Cyber Green PCR Master Mix (Life Technologies, Carlsbad, CA) on a Step One Plus Real-Time PCR System (Life Technologies, Carlsbad, CA). The thermal cycle parameters were an initial hold at $95^{\circ} \mathrm{C}$ for 10 min, 40 cycles of $15 \mathrm{sec}$ at $95^{\circ} \mathrm{C}$ (denature) and $60 \mathrm{sec}$ at $60^{\circ} \mathrm{C}$ (anneal and extend). All samples were run in triplicate with $1.0 \mu \mathrm{L}$ of a 1:10 dilution of cDNA template in a $20 \mu \mathrm{L}$ reaction volume. Primer concentrations were $900 \mathrm{nM}$ for all gene targets. The reference gene used for normalization of expression values was Rp49, a ribosomal protein gene from D. melanogaster. 
Statistical analysis was performed on the Cycle Threshold $(\mathrm{Ct})$ values that were calculated by the Step One Plus v2.2 software, and relative transcript levels were determined using the $\Delta \Delta \mathrm{Ct}$ method [72]. The specific primers for RT-qPCR (Table 4) were selected using the Drosophila RNAi Screening Center at Harvard Medical School's FlyPrimerBank (Boston, MA).

\section{Table 4}

Primer sequences used for RT-qPCR experiments.

\begin{tabular}{|c|c|c|c|}
\hline Gene Class & CG \# & Synonym & Primer Sequence (5'-3') \\
\hline $\begin{array}{l}\text { Ribosomal } \\
\text { Protein }\end{array}$ & 7939 & $\operatorname{Rp} 49$ & $\begin{array}{l}\text { F: CGGTTACGGATCGAACAAGCG } \\
\text { R: TTGGCGCGCTCGACAATCT }\end{array}$ \\
\hline $\begin{array}{l}\text { Cuticular } \\
\text { Protein }\end{array}$ & $\begin{array}{l}11650 \\
4784\end{array}$ & $\begin{array}{c}\text { Lcp } 1 \\
\text { Cpr72Ec }\end{array}$ & $\begin{array}{l}\text { F: CACACCTCCAACGGAATCGA } \\
\text { R: ACTTAACCTCGACGTGCTCG } \\
\text { F: CACTGCTTCACCTGGACGATT } \\
\text { R: CTCCTGGTAACCACTGCTCG }\end{array}$ \\
\hline P450 & $\begin{array}{l}3972 \\
8453 \\
30489 \\
10248 \\
8859 \\
9438\end{array}$ & $\begin{array}{l}\text { Cyp4g1 } \\
\text { Cyp6g1 } \\
\text { Cyp12d1 } \\
\text { Cyp6a8 } \\
\text { Cyp6g2 } \\
\text { Cyp6a2 }\end{array}$ & $\begin{array}{l}\text { F: CCCCACCTTCCACCAGAGCAT } \\
\text { R: AAGTTCTTATTACGATCCTTGACCACC } \\
\text { F: GGTTCCACAACCGATACGC } \\
\text { R: TCCCTGACGAAGAACAGGTTAT } \\
\text { F: TATCTGGCCACGTCGTGATG } \\
\text { R: GCCCCTGGGTTGCATAAAGA } \\
\text { F: CGTTGCACCGCATCTCATC } \\
\text { R: GCCTTGCTTTCCCTGAATTTGTT } \\
\text { R: GCCTTGCTTTCCCTGAATTTGTT } \\
\text { R: CTGATTTCGCGTACTCCCATT } \\
\text { F: CGGGTGATGCACGACTTCTT } \\
\text { R: GTAAAAGCCCACGAAGGGAAA }\end{array}$ \\
\hline ABC B-type & $\begin{array}{l}3879 \\
8523 \\
10181 \\
11897 \\
7806 \\
5789\end{array}$ & $\begin{array}{c}M d r 49 \\
M d r 50 \\
M d r 65 \\
\text { N/A } \\
\text { N/A } \\
\text { N/A }\end{array}$ & $\begin{array}{l}\text { F: TCCCGCAGAGAATACGGGT } \\
\text { R: GAGCCATGCACAGGTAGATGA } \\
\text { F: AGAAGTCCAAGCATGACGAGT } \\
\text { R: CGCTCGATCCTTTTGGTGG } \\
\text { F: AGAGCCTATTGCATTCCTGAAAC } \\
\text { R: GCAGCACATTATGAAGCCGAA } \\
\text { F: GACGAGGAGCTGTATCAACATAG } \\
\text { R: CCTCCCACAGTTCCGCAAAT } \\
\text { F: GCCATATCCGCCTACAATTTCG } \\
\text { R: AGATCCGCCGCAAACAGTT } \\
\text { F: AATACGGATGACCTGACACAATG } \\
\text { R: CGTTTCTGAGATGCGGCTTCT }\end{array}$ \\
\hline ABC C-type & 6214 & Mrpl & $\begin{array}{l}\text { F: AATCGAAAGTATGGCGTGCAG } \\
\text { R: GGGGAATCGACAGCACAGT }\end{array}$ \\
\hline $\begin{array}{l}\text { Glutathione } S \text { - } \\
\text { Transferase }\end{array}$ & $\begin{array}{l}17530 \\
17527\end{array}$ & $\begin{array}{l}\text { GstE6 } \\
\text { GstE5 }\end{array}$ & $\begin{array}{l}\text { F: TACGGTTTGGACCCCAGTC } \\
\text { R: ATATTCCGGTGAAAGTTGGGC } \\
\text { F: CGTGCCGTCAAACTCACTCT } \\
\text { R: GCTGCTCCTGACCCGAAAT }\end{array}$ \\
\hline
\end{tabular}




\section{4 dsRNA synthesis for RNAi by injection}

Total RNA was extracted from 91- $R$ flies and cDNA prepared as described above (section 2.3). For the in vitro transcription reactions yielding dsRNA, a T7-PCR protocol was utilized with Advantage HD Polymerase (Clonetech, Mountain View, CA) following the manufacturer's instructions. Gene specific primers containing the T7-Promoter on the 5' end $(\mathrm{X}=$ TAATACGACTCACTATAGGG) were F-XCATCTGGTATGGTGTGACGC; RXTCCGTATCGGATATTCTCGC for $M d r 49$, F-XTAATTtCCGGAATCTGCTCG; RXGCCGAAAAAGTGGTCATGTT for Mdr50, F-XCGAACTTCTTCCGCACTTTC; RXCATAAAAGGGCCAACTTCCA for Mdr65, and F-XTGGAACAAGCTGAACGTGAG; RXTCAGGTCCCACAGATCCTTC) for Mrpl, respectively. The PCR product was gel-extracted and purified using the QIAquick Gel Extraction Kit (Qiagen, Valencia, CA) and used as a template in the dsRNA synthesis reaction using the MEGAscript T7 transcription kit (Life Technologies, Carlsbad, CA) according to manufacturer's instructions. The resulting dsRNA was purified with a lithium-chloride precipitation step and re-suspended in $30 \mu \mathrm{L}$ of Drosophila injection buffer ( $0.1 \mathrm{mM}$ sodium phosphate, $\mathrm{pH} 6.8 ; 5 \mathrm{mM} \mathrm{KCl})$. To ensure proper dsRNA formation and elimination of persistent secondary structure, the resulting dsRNA was diluted to 1 $\mu \mathrm{g} / \mu \mathrm{L}$ and an annealing cycle was run on the thermal cycler by first incubating the sample at $95^{\circ} \mathrm{C}$ for $3 \mathrm{~min}$, followed by sequential 5 min cooling steps at $85^{\circ} \mathrm{C}, 80^{\circ} \mathrm{C}, 75^{\circ} \mathrm{C}, 70^{\circ} \mathrm{C}, 65^{\circ} \mathrm{C}$, $60^{\circ} \mathrm{C}, 50^{\circ} \mathrm{C}, 40^{\circ} \mathrm{C}, 30^{\circ} \mathrm{C}$, before cooling to $4^{\circ} \mathrm{C}$.

The purified and annealed dsRNA was electrophoresed on a $0.7 \%$ agarose gel with 1.0 $\mu \mathrm{L}$ of a $10 \mathrm{mg} / \mathrm{mL}$ ethidium bromide solution to determine its purity and confirm the expected

product size. $200 \mathrm{~nL}$ of the dsRNA was injected using the Nanoliter 2000 injector (World 
Precision Instruments, Sarasota, FL) into the abdomen of an anesthetized 3 day old adult female fly, which was approximately the same age as 14 days post oviposition.

\subsection{Mortality bioassays}

Ten female flies (14 days post oviposition) per biological replicate ( 3 total) were placed into a $20 \mathrm{~mL}$ glass scintillation vial that was pre-coated with $12.5 \mu \mathrm{g}$ of DDT and capped with cotton moistened with $1 \mathrm{~mL}$ of a $5 \%(\mathrm{w} / \mathrm{v})$ sucrose solution. Females were observed for $24 \mathrm{~h}$ and were considered dead when all movement and leg twitching had ceased. Log time versus logit percent mortality regression lines were generated in order to determine the median lethal time $\left(\mathrm{LT}_{50}\right)$ for the treated flies using the statistical software PoloPC (LeOra, Petaluma, CA). The maximum-log likelihood test was used to determine whether the resulting mortality curves (slope and Y-intercepts) from differently treated fly groups were statistically different $(p<0.05)$.

\section{$\underline{\text { 2.6 Cuticular hydrocarbon analysis }}$}

Groups of 15 females from either the day 9 NHS or day 9 HS F1 progeny were surfaceextracted with n-hexane and the cuticular hydrocarbon differences between the two treatments determined for three replicate experiments using the gas-liquid chromatography (GLC) method described by Strycharz et al. [13]. Structural identification by gas chromatography-mass spectrometry (GC/MS) was carried out on the five cuticular hydrocarbon peaks that were previously determined to be significantly more abundant in the $91-R$ versus the $C S$ flies [13]. 


\subsection{Sequencing of $C y p 6 a 2$}

Genomic DNA (gDNA) was extracted from groups of 15 females from either the $C S$ (DDT-susceptible), 91-C (slightly DDT-resistant), 91-R (highly DDT-resistant), or UAS-Cyp6a2 (the inducible Cyp6a2 RNAi) lines using the DNeasy Blood and Tissue Kit from Qiagen (Valencia, CA). Briefly, females were placed into $2 \mathrm{~mL}$ microcentrifuge tubes containing $180 \mu \mathrm{L}$ Buffer ATL, $0.9 \mu \mathrm{L}$ reagent DX and five stainless steel grinding balls $(0.25 \mathrm{~g}$, SPEX Sample Prep, Metuchen, NJ) and homogenized in an automated homogenizer (GenoGrinder 2010, SPEX Sample Prep, Metuchen, NJ) at 1250 strokes per min for $1 \mathrm{~min}$. Proteinase K $(20 \mu \mathrm{L})$ was added to the tube and incubated for $3-24 \mathrm{~h}$ at $56^{\circ} \mathrm{C}$. Following incubation, the lysate was transferred to a $1.5 \mathrm{~mL}$ tube with $100 \%$ ethanol $(200 \mu \mathrm{L})$ and Buffer AL $(200 \mu \mathrm{L})$ and vortexed. The sample was transferred to a DNeasy Mini spin column (Qiagen), and centrifuged for $30 \mathrm{~s}$ at $6000 \mathrm{~g}$. The flow through was discarded and the column placed into a new collection tube before washing with $500 \mu \mathrm{L}$ buffer AW1, followed by $500 \mu \mathrm{L}$ buffer AW2 with centrifuging for $30 \mathrm{~s}$ at $20,000 \mathrm{~g}$ at each wash. The column was dried by centrifugation for 3 min at 20,000g before DNA elution. The column was placed into a new collection tube and incubated for 1 min with $50 \mu \mathrm{L}$ buffer $\mathrm{AE}$ and centrifuged for $1 \mathrm{~min}$ at $6,000 \mathrm{~g}$ to elute the DNA. This step was repeated to maximize total DNA yield.

DNA was quantified by applying $1 \mu \mathrm{l}$ of extracted gDNA from a single sample to a NanoDrop ND 8000 UV-Vis spectrophotometer (NanoDrop Technologies, Wilmington, DE), absorbance at $260 \mathrm{~nm}$ determined and used to calculate the DNA concentration in ng of DNA $\mu \mathrm{L}^{-1}$ using a modified Beer-Lambert equation. DNA purity was also determined by the ratio 
between absorbencies at 260 and $280 \mathrm{~nm}$, with ratios between 1.5 and 1.8 indicative of pure samples. Samples were diluted to a concentration of $10 \mathrm{ng} \mathrm{DNA} / \mu \mathrm{L}$ and stored at $-20^{\circ} \mathrm{C}$.

The Cyp6a2 sequence was downloaded from NCBI (http://www.ncbi.nlm.nih.gov/ nuccore/U78088.1) and was used as the template for primer design (Fig. 4). $15 \mu \mathrm{L}$ of a master mix $\left(12 \mu \mathrm{L}\right.$ ddH $_{2} \mathrm{O}, 5 \mu \mathrm{L} 5 \mathrm{X}$ Advantage HD Buffer, $1.75 \mu \mathrm{L} 2.857 \mathrm{mM}$ dNTP mixture, $0.25 \mu \mathrm{L}$ Advantage HD Polymerase and $1 \mu \mathrm{L}$ of a $10 \mathrm{mM}$ solution containing the forward and reverse primers (F-GCCTGCATATTATCGCCGAG, and R-ACTAGTCAGGTGGCGATTCG) were mixed with $5 \mu \mathrm{L}$ of template DNA $(10 \mathrm{ng} / \mu \mathrm{L})$ and placed onto an Eppendorf Mastercycler Pro (Hamburg, Germany) thermal cycler operated using the following parameters: 1 cycle at $95^{\circ} \mathrm{C}$ for $1 \mathrm{~min} ; 30$ cycles of $98^{\circ} \mathrm{C}$ for $10 \mathrm{sec}, 60^{\circ} \mathrm{C}$ for $10 \mathrm{sec}, 72^{\circ} \mathrm{C}$ for $2 \mathrm{~min}$; and $2 \mathrm{~min}$ at $72^{\circ} \mathrm{C}$. Gel electrophoresis $(0.9 \%$ agarose) was used to verify quality and quantity of PCR DNA fragment amplification using ethidium bromide ( $1 \mu \mathrm{L}$ of $10 \mathrm{mg} / \mathrm{mL}$ stock) staining to visualize PCR products under UV light.

The QIAquick PCR Purification Kit (Qiagen) was used for PCR product purification following the manufacturer's instructions. Briefly, 5 volumes of buffer PB were added to 1 volume PCR product, mixed with $10 \mu \mathrm{L}$ of $3 \mathrm{M}$ sodium acetate buffer, transferred to a QIAquick spin column placed in a $2 \mathrm{~mL}$ collection tube and centrifuged for $30 \mathrm{~s}$ at $20,000 \mathrm{~g}$. The column was first washed with $750 \mu \mathrm{L}$ guanidine $\mathrm{HCl}(30 \mathrm{~s})$ and then with $750 \mu \mathrm{L}$ buffer PE (30 s). After drying by centrifugation, DNA was eluted into a $1.5 \mathrm{~mL}$ tube by applying $50 \mu \mathrm{L}$ and then $30 \mu \mathrm{L}$ of Buffer EB and centrifuging at 20,000g for $1 \mathrm{~min}$. Gel electrophoresis ( $0.9 \%$ agarose) was used to verify quantity and quality of products as above. 
Triplicates for each sample containing $5 \mu \mathrm{L}$ of a $5 \mu \mathrm{M}$ sequencing primer solution (F1GCGTCGCAGGGGAATCTTAT, F2-AGCACCTGTTCAACCTGGAC, F3 ACGACT TCAT GA A C C T GCTG, F 4-ACA GGTCATAAT C C C CG C T T, R 1 TGAACCTGCAAATCTGATGGG，R2-TGGGATCTCTGTCGTATCGC，R3G T T C C T C C A GCACC G T T T G, R 4 - T CA GC T C C T T G ATC T C G A GC, R 5 CCGACCATGTTGCCATACAG) were mixed with $10 \mu \mathrm{L}$ of purified PCR product at a concentration of $(2 \mathrm{ng} / \mu \mathrm{L})$, loaded into a 96 well plate and sent to GeneWiz (South Plainfield, NJ) for sequencing. Sequence chromatograms were analyzed using Chromas lite software (Technelysium Pty Ltd., Tewantin, Australia) to determine sequences, which were then loaded into CLC Sequence viewer (Boston, MA) for sequence alignment and protein translation. 
GCCTGCATATTATCGCCGAGTCTGTAATCATGACAACAACTTAAAAGTAGTAGTCA TGGTGATAGAAATATTTAGCTAGCTAGCTCACATGCTGTCATGCCTGTGCGTCGCAG GGGAATCTTATAAAAAGTGTGCGAACATATTGTGGTGATCAGTAATTCGTCGTAGGT CGAGCACGACGATTGCGAAAAGGGAGCAGCTACGCAAAATGTTTGTTCTAATATAC CTGTTGATCGCGATCTCCTCGCTTTTGGCCTACTTGTACCACCGCAACTTCAACTACT GGAATCGCCGCGGCGTGCCACACGATGCTCCTCACCCACTGTATGGCAACATGGTC GGGTTCCGGAAGAACCGGGTGATGCACGACTTCTTCTACGACTACTACAACAAGTA CCGGAAGAGCGGCTTTCCCTTCGTGGGCTTTTACTTTCTGCACAAGCCGGCCGCCTT CATCGTGGACACCCAGCTGGCCAAGAACATCCTGATCAAGGATTTCTCGAACTTTG CCGATCGTGGCCAGTTTCACAACGGGCGCGACGACCCGCTCACGCAGCACCTGTTC AACCTGGACGGAAAGAAGTGGAAGGACATGCGCCAGAGGCTGACGCCGACTTTCA CCTCGGGCAAGATGAAGTTCATGTTCCCGACGGTGATCAAGGTGTCTGAGGAGTTC GTCAAGGTGATCACGGAGCAGGTGCCCGCCGCCCAGAACGGCGCTGTGCTCGAGA TCAAGGAGCTGATGGCCAGGTTCACCACCGATGTGATTGGCACCTGTCGCTTCGGC ATTGAGTGTAACACGCTGCGCACCCCTGTCAGTGATTTCCGCACCATGGGACAGAA GGTGTTCACCGATATGCGCCACGGGAAACTGCTGACCATGTTCGTGTTCAGCTTTCC CAAGCTGGCCAGCAGGTTGAGAATGCGCATGATGCCCGAGGACGTCCACCAGTTCT TCATGCGCCTGGTCAACGACACGATTGCCCTCAGGGAGCGGGAGAACTTCAAGAG GAACGACTTCATGAACCTGCTGATTGAACTGAAGCAGAAGGGGCGCGTCACCCTG GACAACGGAGAGGTGATCGAGGGCATGGACATCGGCGAACTGGCCGCCCAGGTGT TCGTCTTTTATGTGGCCGGATTTGAGACCTCCTCCTCGACAATGAGTTACTGCCTGTA TGAGTTGGCTCAGAATCAGGACATTCAGGACAGGCTGCGCAACGAGATCCAAACG GTGCTGGAGGAACAGGAGGGGCAGCTAACGTACGAATCCATCAAAGCCATGACCTA CTTGAACCAGGTCATCTCAGGTAGGTGTTCTGTCTGAAGCACAGCCCCAAGAGGGATT GTAACTGGTTTTACCCAACATTGCTCTTCAGAAACCCTGAGGCTCTACACACTGGTGCC CCACCTCGAACGGAAGGCCCTCAACGACTACGTGGTGCCGGGCCATGAAAAGCTT GTGATTGAGAAGGGCACACAGGTCATAATCCCCGCTTGCGCCTACCACCGCGACGA GGATCTTTATCCGAATCCGGAGACCTTTGATCCGGAGCGCTTCTCGCCGGAGAAAGT GGCCGCCCGGGAGTCCGTGGAGTGGCTGCCCTTCGGCGACGGGCCGCGGAACTGC ATCGGGATGCGGTTTGGACAAATGCAGGCTCGCATCGGTTTGGCTCAGATCATCAGC CGGTTCAGGGTATCCGTCTGCGATACGACAGAGATCCCACTGAAGTATAGTCCCATG TCCATAGTTTTGGGCACCGTTGGGGGCATCTACTTGCGAGTGGAACGCATCTAACCT CCATATTCGTTGCTCCCATGTATATAGCTTAGGATCCAAAGCTAAAGTGATGTACATTT TAGACTGTTCAATTATTAAATAACCTTAACCTAAACAGCCATATTAACTTATTGGCCT GTGATAAATCACTTATGTTCACAGTTTAGAAGATCTAATTGTCTACCAGTTAGATGCA TTCAGGCAAATGTTGTTCCCATCAGATTTGCAGGTTCATAAATTGCATTCGGCTTAAG TTGCAACATGCCACGCGAATCGCCACCTGACTAGT

Figure 4. The primer design for Cyp6a2 sequencing. Blue text represents forward primers, while red text represents reverse primers. The bold primers are those used for the PCR amplification, while non-bolded primers were used for the sequencing reaction. The start and stop codons are underlined, while the only intron has been italicized. 


\section{CHAPTER 3}

\section{ROLE OF UAS-RNAi VALIDATED GENES IN DDT SENSITIVITY AND RESISTANCE $\underline{\text { 3.1 Role of cuticular genes in DDT sensitivity and resistance }}$}

In order to establish that the Cyp4g1 gene was over transcribed by $91-R$ versus $C S$ flies, RT-qPCR analyses were carried out (Fig. 5). The expression level of Cyp4g1 was significantly increased 1.4-fold in 91-R flies compared with $C S$ flies. This finding correlates to the increased abundance of cuticular hydrocarbons associated with the epicuticle layer in $91-R$ flies and may be responsible, in part, for the decreased rate of DDT penetration when topically applied [13]. Three candidate cuticular genes, Cyp4g1, Lcp 1, and Cpr72Ec, were then selected for UAS-RNAi testing using the criteria described in Table 2.

To determine the most appropriate time for heat shock-induced RNAi experiments, a time course experiment was carried out where F1 progeny from crosses of the Driver 1799 and UASRNAi line 102864 (Cyp4gl) were either non-heat shocked (NHS, control) or heat shocked (HS) at 3,6 , or 9 days post oviposition. The same 5 hydrocarbon peaks that were significantly more abundant in the 91-R strain compared with $C S$ strain (peak 1, 9-triacosene; peak 2, triacosane; peak 3, pentacosane; peak 4, heptocosadiene; peak 5, heptocosane) [13], were analyzed for abundance changes in the HS versus the NHS groups $(p<0.05)$ (Fig. 6). No significant differences in the five hydrocarbon peaks were found between the NHS and HS flies at either 3 or 6 days post oviposition. Following HS at 9 day post oviposition, hydrocarbon peaks 1, 3, 4, and 5 were all significantly reduced compared to the corresponding NHS flies $(\mathrm{p}<0.05)$. Peak 1 was reduced by $94 \%$, peak 3 by $83 \%$, peak 4 by $88 \%$, and peak 5 by $86 \%$ (Fig. 6). Peak 2 was also reduced but not significantly. 


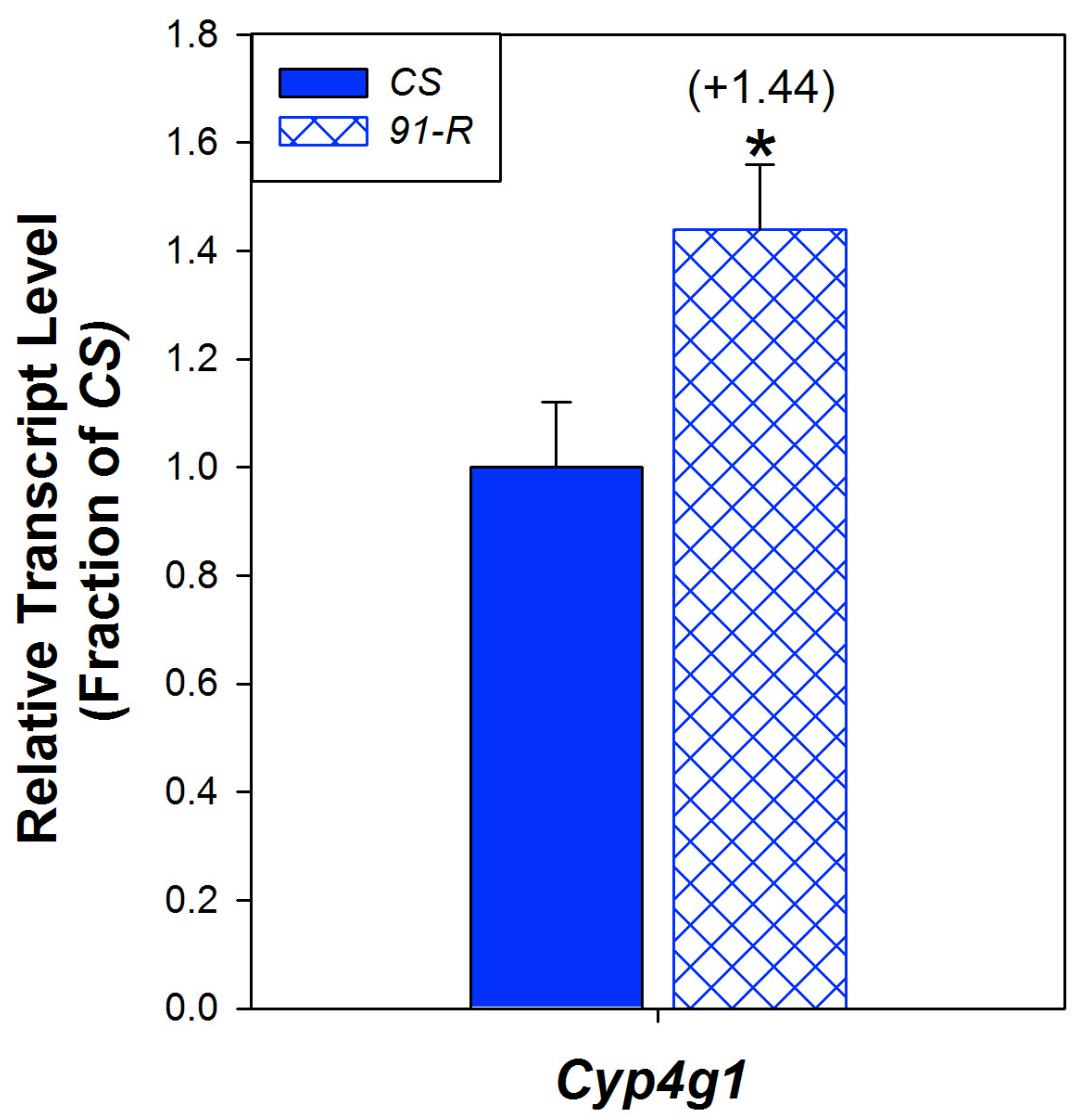

Figure 5. Cyp4gl was over transcribed in the DDT-resistant $91-R$ strain compared to the insecticide-susceptible $C S$ strain. Relative transcript levels were measure by RT-qPCR and analyzed using the $\triangle \triangle \mathrm{CT}$ method with mean values \pm S.D. of 3 biological replicates reported. The value in parentheses indicated the fold transcript increase over the $C S$ strain. A single asterisks $\left(^{*}\right)$ indicates a statistically significant increase in the transcript level using Student's $t$ test $(\mathrm{p}<0.05)$. 


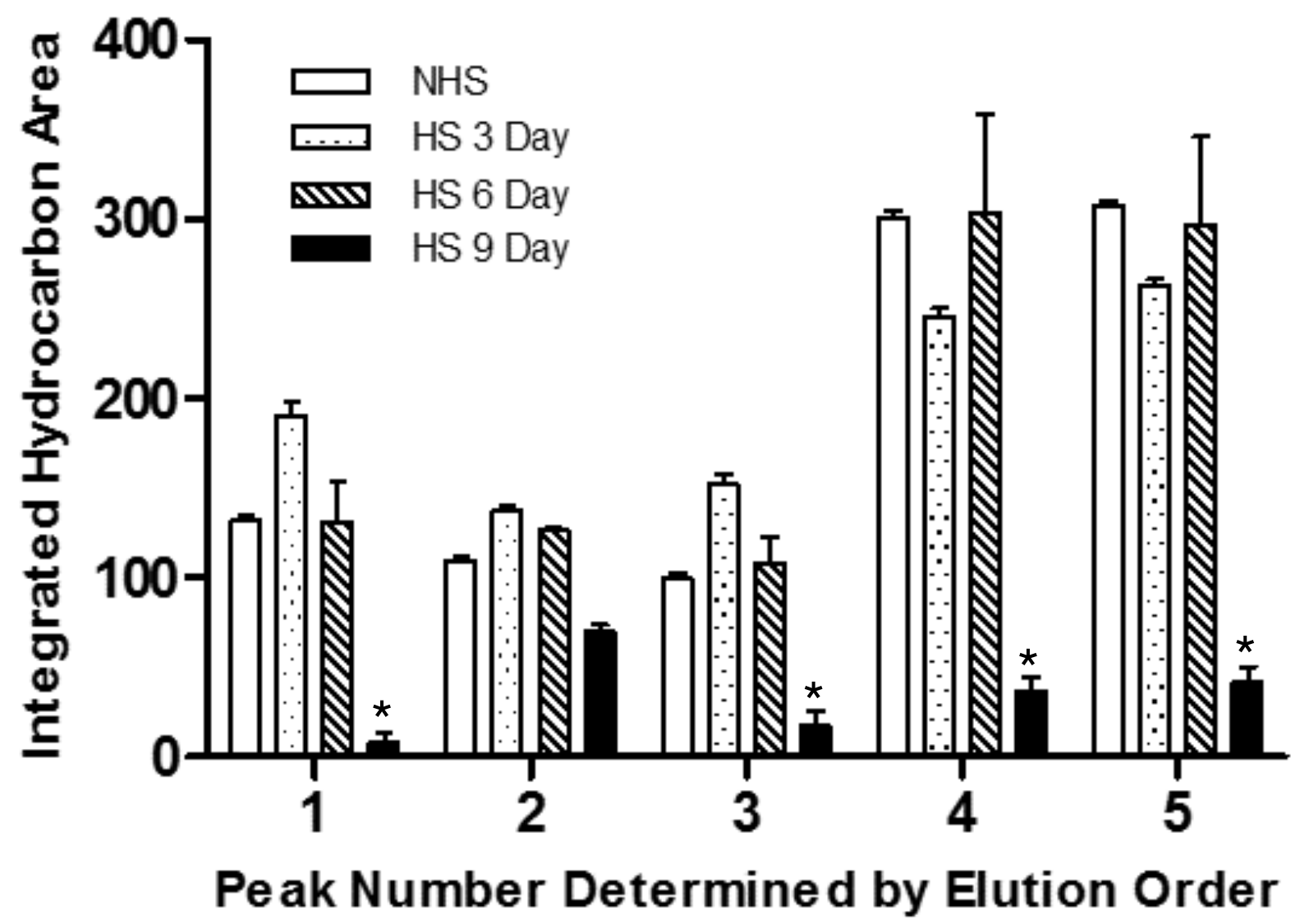

Figure 6. Cuticular hydrocarbon analyses by GC-FID of 3 biological replicates ( 15 females each) of crosses between Driver 1799 and UAS-RNAi line 102864 (Cyp4g1). Samples were either non-heat shocked (NHS), or heat shocked (HS) at 3, 6, or 9 days post oviposition. A grouped, two-way ANOVA analysis was performed to determine the significance of hydrocarbon peak reduction seen between HS versus NHS F1 females using S.E.Ms. A single asterisk $(*)$ indicates statistically significant reduction in hydrocarbon abundances after HS induced RNAi compared with the NHS flies $(\mathrm{p}<0.05)$. 
Following the establishment of a heat shock protocol (9 days post oviposition), crosses between the heat shock-inducible Gal4 Driver 1799 and the UAS-RNAi lines containing the inverted gene repeats for the 3 genes putatively involved in reduced cuticular penetration of DDT (Cyp4g1, Lcpl, and Cpr72Ec) were performed as described above. The F1 progeny were tested by RT-qPCR analysis to confirm gene knockdown and DDT sensitivity was determined by mortality bioassays. For Cyp4g1, Lcpl, and Cpr72EC, the relative transcript levels as determined by RT-qPCR were significant reduced $(\mathrm{p}<0.05)$ by $50 \%(+/-6 \%), 57 \%(+/-9 \%)$, and $48 \%(+/-$ 4\%), respectively, and indicated that the UAS RNAi knockdown strategy was functional (Fig. 7A). In the DDT mortality bioassays, however, increases in susceptibility to DDT following RNAi knockdown were only significant in the Cyp4g1 and Lcp 1 knockdown flies as determined by their respective $\mathrm{LT}_{50}$ values (Fig. 7B). Cyp $4 g 1$ RNAi resulted in a $25 \%$ reduction in the $\mathrm{LT}_{50}$ value ( $\chi 2$; d.f.; Tail Probability, 56.96; $2 ;<0.001$ ), and $L c p 1$ RNAi resulted in a $14 \%$ reduction $(\chi 2$; d.f.; Tail Probability, $16.04 ; 2 ;<0.001)$. These findings indicate that both Cyp4gI and Lcpl are involved in DDT sensitivity and may play a role in decreasing the rate of DDT penetration in resistant flies, perhaps in a synergistic fashion. It is also of interest to note that $91-R$ flies had a significantly thicker cuticle than the $C S$ flies and that the endocuticle of $91-R$ flies was noticeably more laminated [13]. 

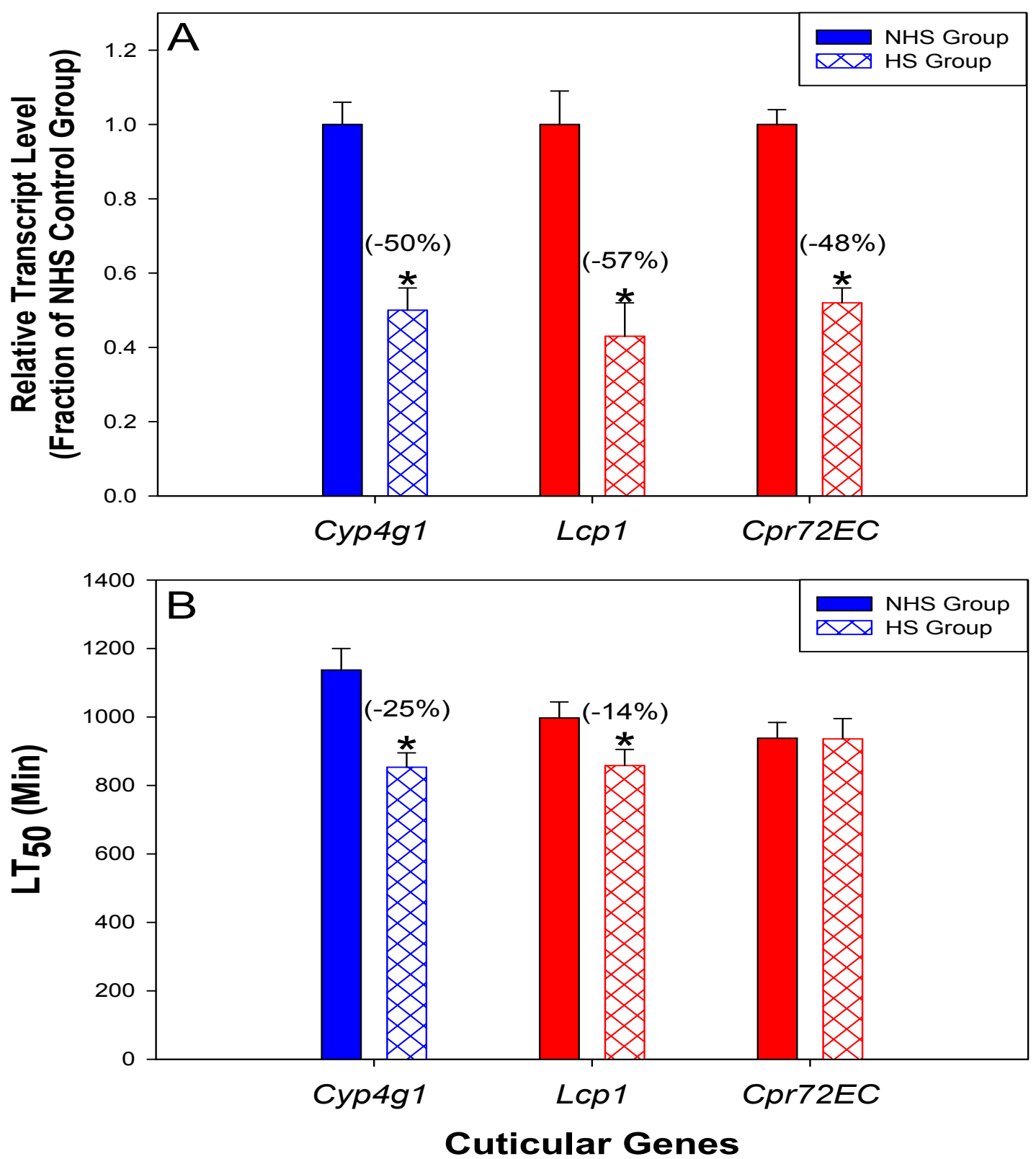

Figure 7. UAS-RNAi qPCR and mortality bioassay validation of genes putatively involved in the penetration of DDT. Following crosses of the Driver 1799 and appropriate UAS-RNAi lines, control NHS F1 females were compared to the day 9 HS RNAi groups. Three biological replicates of $30 \mathrm{~F} 1$ females each at 14 days post oviposition used for RT-qPCR analysis (Panel A). For mortality bioassays, three groups of $10 \mathrm{~F} 1$ females each were used and DDT mortality observations were taken every hour for $24 \mathrm{~h}$ (Panel B). Values in parentheses represent percent reductions in either transcript levels or $\mathrm{LT}_{50}$ values of HS versus NHS groups, respectively. A single asterisk (*) indicates statistically significant reductions in either transcript levels using Students $t$-test in Panel A or in LT $_{50}$ values using the maximum-log likelihood ratio test in Panel B (both at $\mathrm{p}<0.05$ ). 


\subsection{Role of phase I or II metabolism genes in DDT sensitivity and resistance}

Seven candidate genes putatively involved in phase I (Cyp6g1, Cyp12d1, Cyp6a8, Cyp6g2, Cyp6a2) or phase II (GstE5, GstE6) metabolism of DDT were selected for UAS-RNAi testing using the criteria described in Table 2. Crosses between the heat shock-inducible Gal4 Driver 1799 and the UAS-RNAi lines containing the gene knockdown sequences of these genes were performed as described above. The relative transcript levels were significantly reduced $(\mathrm{p}<0.05)$ by $68 \%(+/-4 \%), 59 \%(+/-7 \%), 57 \%(+/-6 \%), 50 \%(+/-5 \%), 63 \%(+/-3 \%) 51 \%(+/-$ 5\%) and 55\% (+/- 3\%) for Cyp6g1, Cyp12d1, Cyp6a8, Cyp6g2, Cyp6a2, GstE5 and GstE6, respectively, indicating successful gene knockdown (Fig. 8A). In the DDT mortality bioassays, however, increases in susceptibility to DDT following RNAi knockdown were only significant in the Cyp6gl and Cyp12d1 knockdown flies as determined by their respective $\mathrm{LT}_{50}$ values (Fig. 8B). Cyp6g1 RNAi resulted in a $16 \%$ reduction in the $\mathrm{LT}_{50}$ value ( $\chi 2$; d.f.; Tail Probability, 22.00; $2 ;<0.001)$, and Cyp12d1 RNAi resulted in a 10\% reduction ( $\chi 2$; d.f.; Tail Probability, 9.54; $2 ; 0.008)$. These findings indicate that both Cyp6g1 and Cyp12d1 are involved in DDT sensitivity in the transgenic flies and may play a role in the metabolism of DDT and perhaps in resistance. Of note is the finding that one of the major metabolites formed from DDT by $91-R$ flies was dicofol, its hydrolytic breakdown product, dichlorobenzophenone, and their respective water-soluble conjugates [13]. Interestingly, Cyp12d1 in $91-R$ flies has a premature stop codon and therefore is not involved in the oxidative metabolism of DDT [15] and suggests a major role of Cyp6gl in the oxidative metabolism and detoxification of DDT in the $91-R$ strain. 

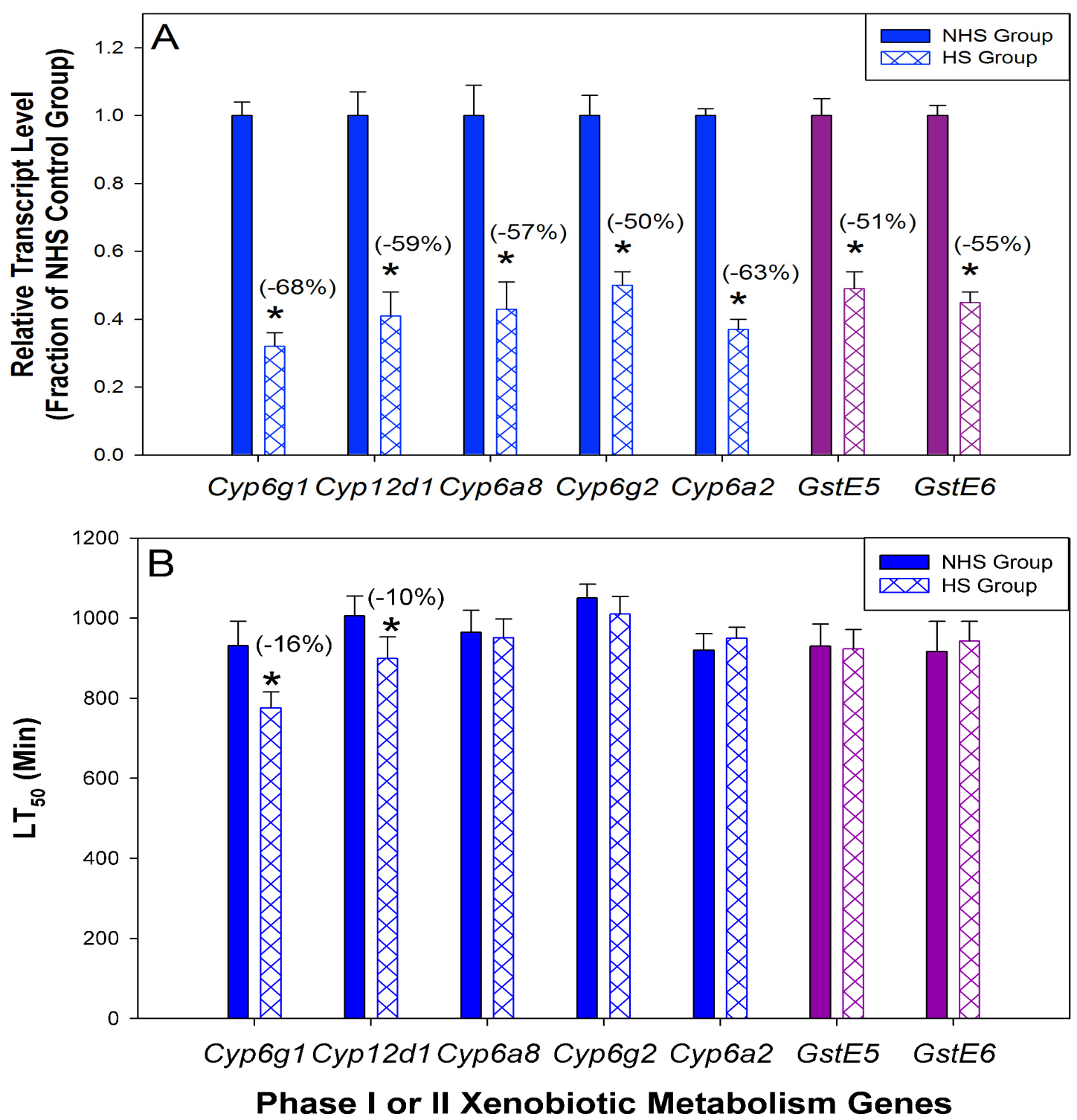

Figure 8. UAS-RNAi qPCR and mortality bioassay validation of genes involved in the phase I or II xenobiotic metabolism of DDT. Following crosses of the Driver 1799 and appropriate UAS-RNAi lines, control NHS F1 females were compared to the day 9 HS RNAi F1 females. Three biological replicates of $30 \mathrm{~F} 1$ females each at 14 days post oviposition were used for RTqPCR analysis (Panel A). For mortality bioassays, three groups of 10 female flies each were used and DDT mortality observations were taken every hour for $24 \mathrm{~h}$ (Panel B). Values in parentheses represent percent reductions in transcript levels or $\mathrm{LT}_{50}$ values of HS versus NHS groups, respectively. A single asterisk $\left({ }^{*}\right)$ indicates statistically significant reductions in either the transcript levels using Students $t$-test in Panel A or in $\mathrm{LT}_{50}$ values as determined by the maximum-log likelihood ratio test in Panel B (both at $\mathrm{p}<0.05$ ). 
GstE5 and GstE6 were also determined to be over transcribed in 91-R flies and are hypothesized to provide indirect protection by scavenging reactive oxygen species (ROS) produced during the stress caused by DDT exposure and metabolism [15]. The lack of DDT effect in mortality bioassays using the respective heat shocked transgenic flies may be that these genes are simply not expressed or expressed only at low levels. An alternative explanation is that GstE5 and GstE6 are simply not needed in the insecticide-susceptible UAS-RNAi flies because they are not metabolizing DDT to the same extent as seen in the $91-R$ flies. Nevertheless, they still may be necessary to offset the ROS generation through constitutive over expression of P450s seen in the $91-R$ strain. It is possible therefore that RNAi of these genes in the DDTresistant $91-R$ strain may result in increases in the susceptibility to DDT due to impairment of the GSTs ability to offset ROS production, even though increases in susceptibility were not seen in the insecticide-susceptible UAS-RNAi flies. 


\subsection{Role of phase III metabolism genes in DDT sensitivity and resistance}

Seven candidate genes involved in phase III metabolism, Mdr49, Mdr50, Mdr65, Mrp1, CG11817, CG5789, and CG7806, were selected for UAS-RNAi testing using the criteria described in Tables 2. Crosses were performed and tested as before. Relative transcript levels were significantly reduced $(\mathrm{p}<0.05)$ by $58 \%(+/-5 \%), 49 \%(+/-3 \%), 61 \%(+/-4 \%), 54 \%(+/-$ 6\%), 59\% (+/- 5\%), 52\% (+/- 7\%), and 56\% (+/- 4\%) for Mdr50, Mdr65, Mdr49, Mrp1, CG11817, CG5789, and CG7806, respectively, indicating successful gene knockdown (Fig. 9A). In the DDT mortality bioassays, however, increases in susceptibility to DDT following RNAi knockdown were only significantly increased in the $M d r 50, M d r 65$, and $M r p 1$ knockdown flies as determined by their respective $\mathrm{LT}_{50}$ values (Fig. 9B). Mdr50 RNAi resulted in a $13 \%$ reduction in the $\mathrm{LT}_{50}$ value ( $\chi 2$; d.f.; Tail Probability, 16.56; $2 ;<0.001$ ), Mdr65 RNAi resulted in a $12 \%$ reduction ( $\chi 2$; d.f.; Tail Probability, $11.94 ; 2 ; 0.002$ ), and Mrp1 RNAi resulted in a $15 \%$ reduction ( $\chi 2$; d.f.; Tail Probability, $23.70 ; 2 ;<0.001)$. These findings indicate that both ABC Band C-types of $\mathrm{ABC}$ transporters are involved in DDT sensitivity in the transgenic flies and may play a role in the ATP-driven efflux of DDT and perhaps in resistance. As shown previously, verapamil, a well-established substrate for ABC B-Type transporters, pretreatment significantly synergized the toxic action of DDT on 91-R flies but had only limited effect on $C S$ flies [13]. Furthermore, 91- $R$ flies effluxed 3.3-fold more unmetabolized DDT than $C S$ flies. 

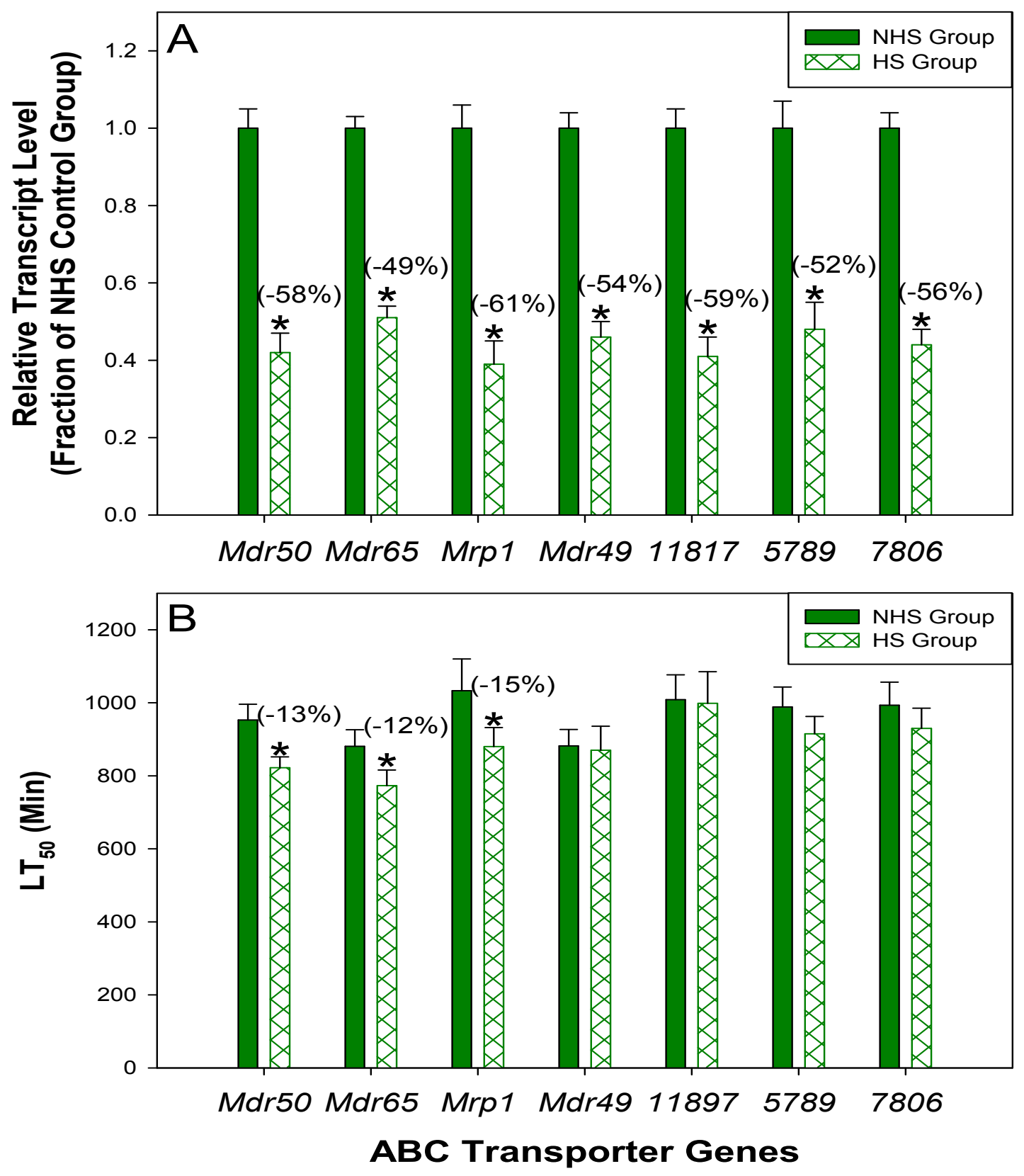

Figure 9. UAS-RNAi qPCR and mortality bioassay validation of genes involved in the phase III metabolism of DDT. Following crosses of the Driver 1799 and appropriate UAS-RNAi lines, control NHS F1 females were compared to the day 9 HS RNAi F1 females. Three biological replicates of $30 \mathrm{~F} 1$ females each at 14 days post oviposition were used for RT-qPCR analysis (Panel A). For mortality bioassays, three groups of 10 female flies each were used and mortality observations were taken every hour for $24 \mathrm{~h}$ (Panel B). Values in parentheses represent either percent reductions in transcript levels or $\mathrm{LT}_{50}$ values of HS versus NHS groups, respectively. A single asterisk (*) indicates statistically significant reductions in either the transcript levels using Students $t$-test in Panel A or in $\mathrm{LT}_{50}$ values as determined by the maximum-log likelihood ratio test in Panel B (both at $\mathrm{p}<0.05$ ). 
In an attempt to knock down the ABC transporters believed to efflux DDT in the $91-R$ strain, intra-abdominal dsRNA injections were performed in anesthetized $91-R$ female flies $[73$, 74]. First, the resulting T7-PCR product was visualized by $(0.9 \%)$ agarose gel with ethidium bromide staining (Fig. 10A). Because there were multiple bands present after the T7-PCR, a gel purification protocol was utilized to extract the band of interest to use as a template in the dsRNA synthesis reaction. After gel extraction purification, the dsRNA was synthesized, purified, annealed, and electrophoresed on a $(0.7 \%)$ formaldehyde agarose gel utilizing ethidium bromide staining for visualization (Fig. 10B). After the RNA synthesis, only a single band was present of the expected size $(\sim 500 \mathrm{bp})$.

After dsRNA injection, however, no decrease in the transcript levels of the $\mathrm{ABC}$ transporters were found in the 91-R line. There are several possible explanations for this, such as inefficient cellular uptake of the dsRNA, or perhaps inefficient dsRNA constructs against the genes of interest. In the future, it would make sense to design a dsRNA that is the same as that expressed by the UAS-RNAi line, in order to determine the reason why the particular dsRNAs that were injected did not have an effect on the transcript level. 


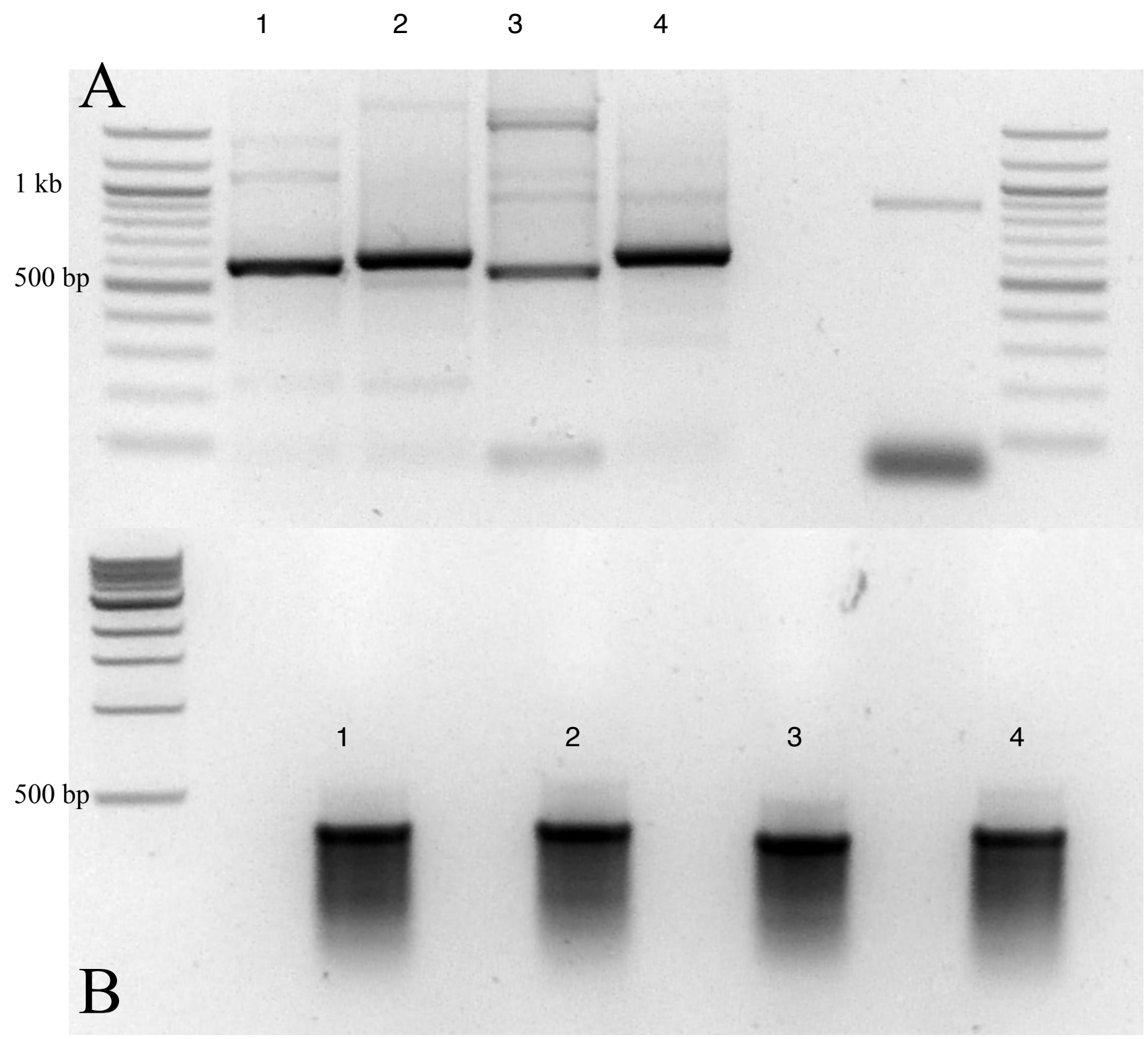

Figure 10. The synthesis of gene specific dsRNAs for intra-abdominal injection induced RNAi against the ABC-Transporters Mdr49 (Lane 1, 553 bp), Mdr50 (Lane 2, 509 bp), Mdr65 (Lane 3, $508 \mathrm{bp}$ ), and Mrpl (Lane 4, $577 \mathrm{bp}$ ) consisted of two steps. Panel A shows the products after PCR amplification using cDNA as template. Due to the presence of multiple bands, the PCR products were gel purified before running the T7 MEGAscript RNA reaction. Panel B shows the resulting dsRNA that was annealed and electrophoresed on a formaldehyde denaturing agarose gel to confirm bands of the expected size were present. 


\section{$\underline{\text { 3.4 Discussion }}$}

Of the 17 genes tested by UAS-RNAi, 7 were found to have a significant effect on susceptibility to DDT as determined by mortality bioassay (Fig. 11). Knockdown of Cyp4g1 was found to increase susceptibility to DDT by $25 \%$ and this gene is located on the X chromosome (chromosome 1); the only sex-linked resistance factor that has been determined to date. Lcp 1, Cyp6g1, Cyp12d1, and Mdr50 are located on chromosome 2R and Mrpl is located on the left $\operatorname{arm}(2 \mathrm{~L})$ of chromosome 2 . UAS-RNAi resulted in a $14 \%, 17 \%, 11 \%, 13 \%$ and $15 \%$ increase in susceptibility to DDT, respectively, making this chromosome prominent in DDT resistance. Mdr65 is located on chromosome $3 \mathrm{~L}$ and its knockdown by UAS-RNAi resulted in a $12 \%$ increase in susceptibility to DDT. Thus, multiple genes across all three chromosomes appear to be involved in DDT resistance in the highly resistant $91-R$ strain. These results are consistent with the original suggestion that DDT resistance in the $91-R$ strain is multifactorial and that factors on all three chromosomes are involved in DDT resistance [8-11]. Factors on chromosome 2 were suggested to play a major role, followed by factor(s) on chromosome 3, with the factor(s) on chromosome 1 showing a much smaller but statistically significant effect [19]. It was also suggested that the factors on chromosomes 2 and 3 interacted in a more than additive manner. Our results therefore suggest that the Cyp4gl gene on chromosome 1 is a likely candidate as the resistance factor associated with this sex chromosome. Also Mdr65 on chromosome 3 is likely a resistance factor and may interact with either the $\mathrm{P} 450$ genes or the other $\mathrm{ABC}$ transporter genes on chromosome 2 in a synergistic fashion, resulting in extremely high levels of resistance. This conjecture, however, will need future experimental proof.

What is not yet known is how these resistance mechanisms interact with each other. Will 
it be in a less than additive, additive, or greater than additive manner? By injecting dsRNA into $91-R$ flies for each of the identified 7 genes individually, and then in combination, we should be able to determine how these multiple resistance mechanisms, found across all three chromosomes, interact. 


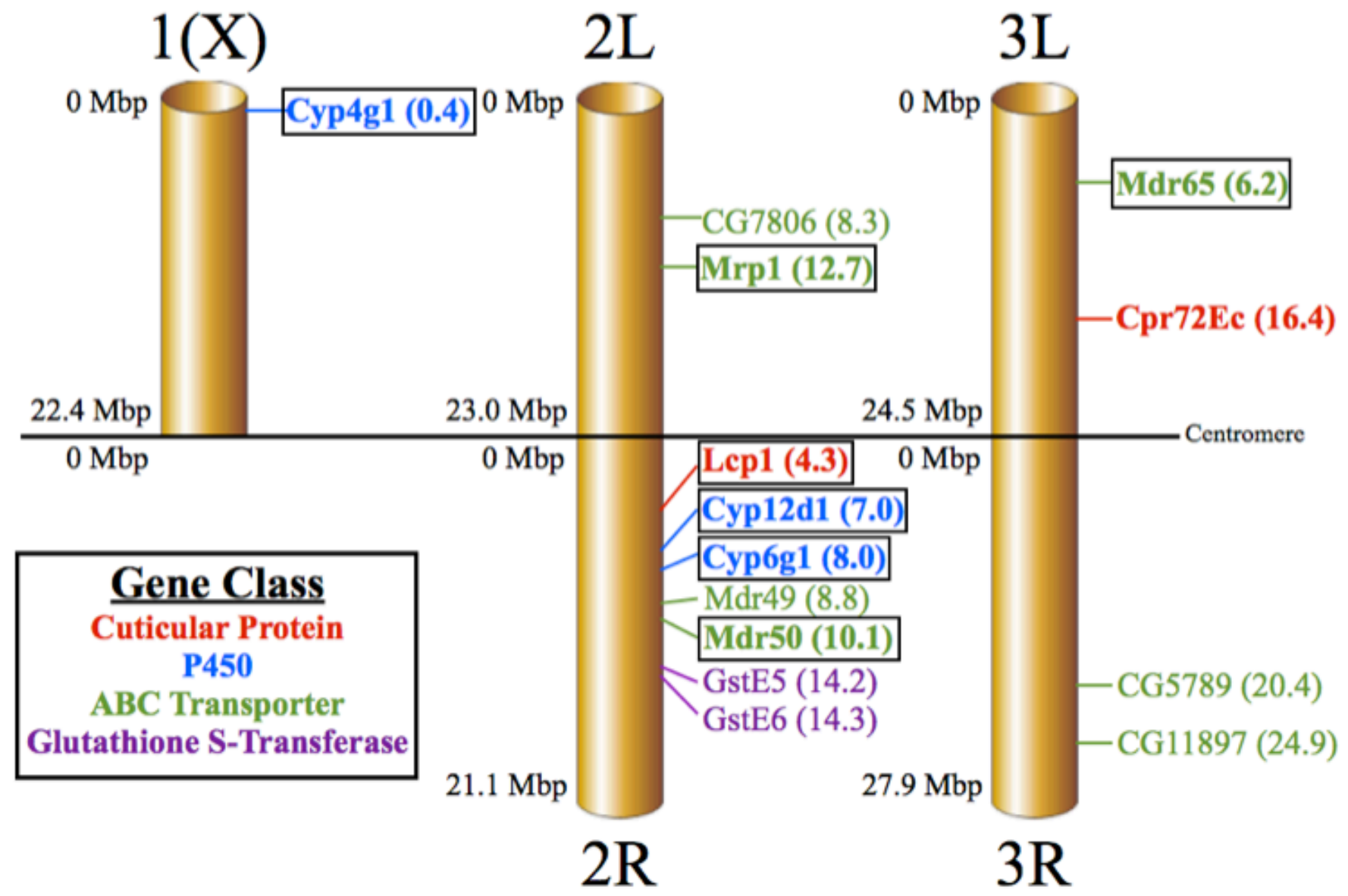

Figure 11. Genes putatively involved in DDT resistance through literature and bioinformatics review validated through use of the UAS-RNAi system (number in parentheses are approximate chromosome locations in mega-basepairs as determined by NCBI Map Viewer). The right arm of chromosome 2 seems to be highly involved in DDT resistance, with 4 of the 7 resistance factors present. 


\section{CHAPTER 4}

\section{SEQUENCING OF CYP6A2}

\subsection{Nucleotide sequence analysis}

In order to determine if the mutations that enabled DDT metabolism found in the Cyp6a2SVL line were present in the highly DDT-resistant 91-R strain, the Cyp6a2 gene was sequenced across several strains of D. melanogaster. These strains included the DDT-susceptible CS, DDT-susceptible UAS-Cyp6a2 line (Cyp6a2 RNAi), slightly DDT-resistant 91-C, highly DDT-resistant 91-R, and the Cyp6a2SVL mutant, which can metabolize DDT [56]. In order to assemble the full length sequence, the five forward and reverse sequencing reactions that spanned the gene were assembled as contigs. In all cases, the individual sequencing reactions overlapped by a minimum of $100 \mathrm{bp}$. The ends were trimmed to include only the highest quality nucleotide signals, and assembled end to end. The end result was a $\sim 2100 \mathrm{bp}$ continuous read length sequence that could be compared between all sequenced lines.

After PCR amplification, the resulting products were run on an agarose $(0.9 \%)$ gel to confirm the bands were of the expected size. While bands were expected to be $2.0 \mathrm{~kb}$ in length based on the NCBI template, the CS, 91-C, and UAS-Cyp6a2 lines all contained PCR products that were about $2.5 \mathrm{~kb}$ in length (Fig. 12). This discrepancy in band size was not due to changes in the coding region of the gene, instead it was due to the insertion of a $\sim 500 \mathrm{bp}$ fragment on the 3 ' region of the gene in the $C S, 91-C$, and UAS-RNAi lines.

In the coding region, there were 21 Single Nucleotide Polymorphisms (SNP's) found (Fig. 13). While many of the SNP's found were present in only 1 of the 5 lines sequences, there were 5 mutations that were found across multiple sequenced lines, implying that these particular 
mutations had been genetically selected for and were not purely random occurrences. At nucleotide position 408, both the 91-R and Cyp6a2SVL strains had T, while the other 3 lines had G. At nucleotide position 679 , the $C S$ and $91-C$ strains had A while the other lines had G. At nucleotide position 916, the $91-R$ and Cyp6a2SVL strains had $\mathrm{G}$ while the other lines had A. At nucleotide position 933 the $91-R$ and Cyp6a2SVL had A while the others had C. At nucleotide position 1301, the 91-R and Cyp6a2SVL had $\mathrm{C}$ while the other lines had A. Of particular note, the $91-C$ line has a base pair deletion at nucleotide position 1341. This deletion leads to a frame shift mutation, likely leading to a non-functional Cyp6a2 gene. The Cyp6a2SVL line had been previously reported to metabolize DDT, however the highly DDT-resistant $91-R$ line did not possess any of the mutations resulting in the Cyp6a2SVL amino acid changes associated with the Cyp6a2SVL line. Instead, our analysis showed additional mutations present that both the highly DDT-resistant 91-R line and Cyp6a2SVL line shared (nucleotide positions 408, 916, 933, and 1301).

Further sequence analysis showed that the $C S, 91-C$, and UAS-Cyp6a2 lines all contained a $500 \mathrm{bp}$ insertion in the 3 ' region of Cyp6a2, while the $91-R$ strain did not contain this insertion (Fig. 14) It is unclear what the exact consequence of this insert may be, although it is likely influential on transcript stability. The $91-R$ line has Cyp6a2 transcript levels much higher than the susceptible lines, and it is possible that this is due in part to the transposon insert. 


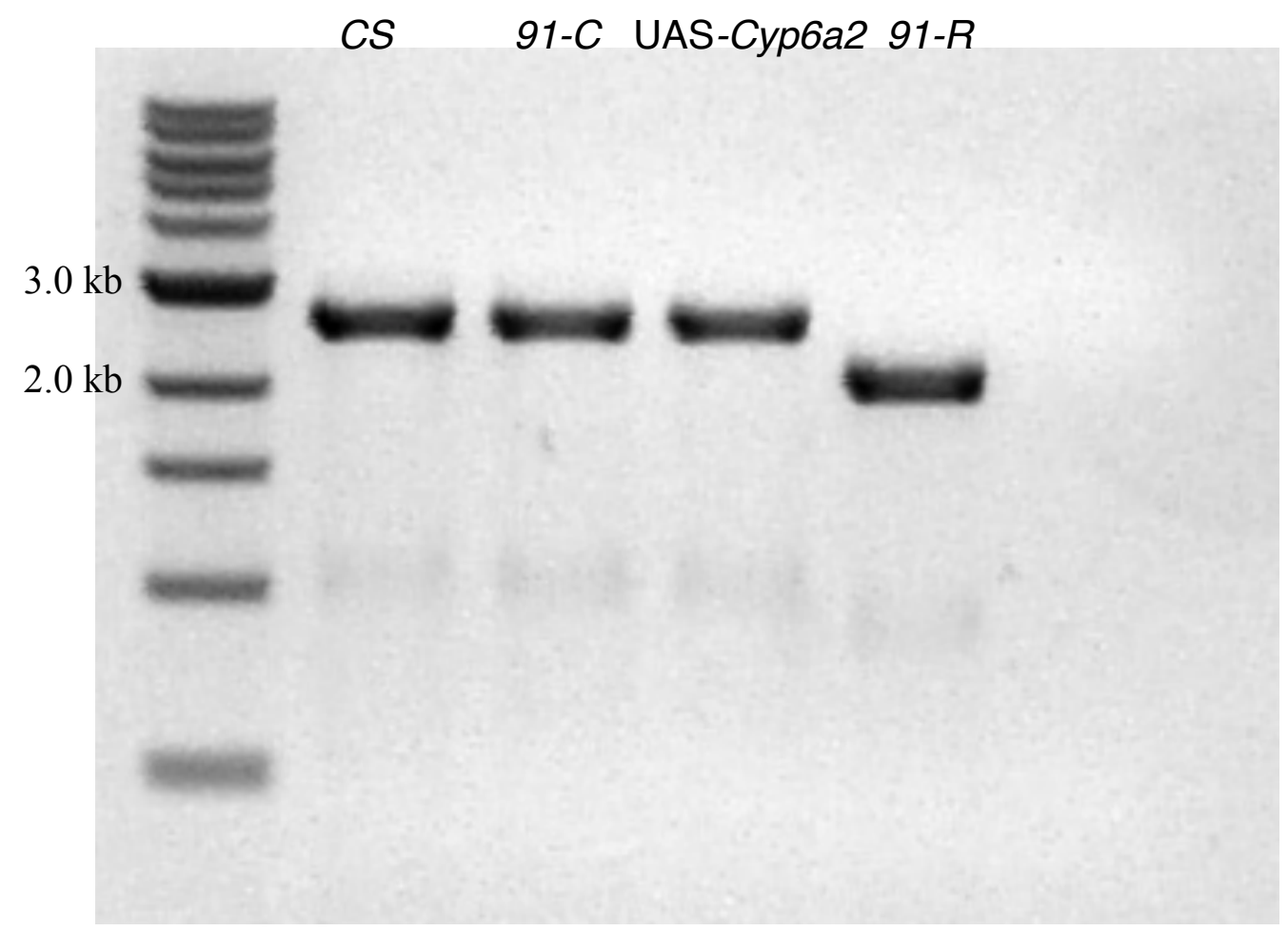

Figure 12. Agarose gel showing the PCR product of Cyp6a2 for the $C S, 91-C$, UAS-Cyp6a2, and $91-R$ strains. The $91-R$ band was about $2.0 \mathrm{~kb}$ while the other strains PCR products were about $2.5 \mathrm{~kb}$. 


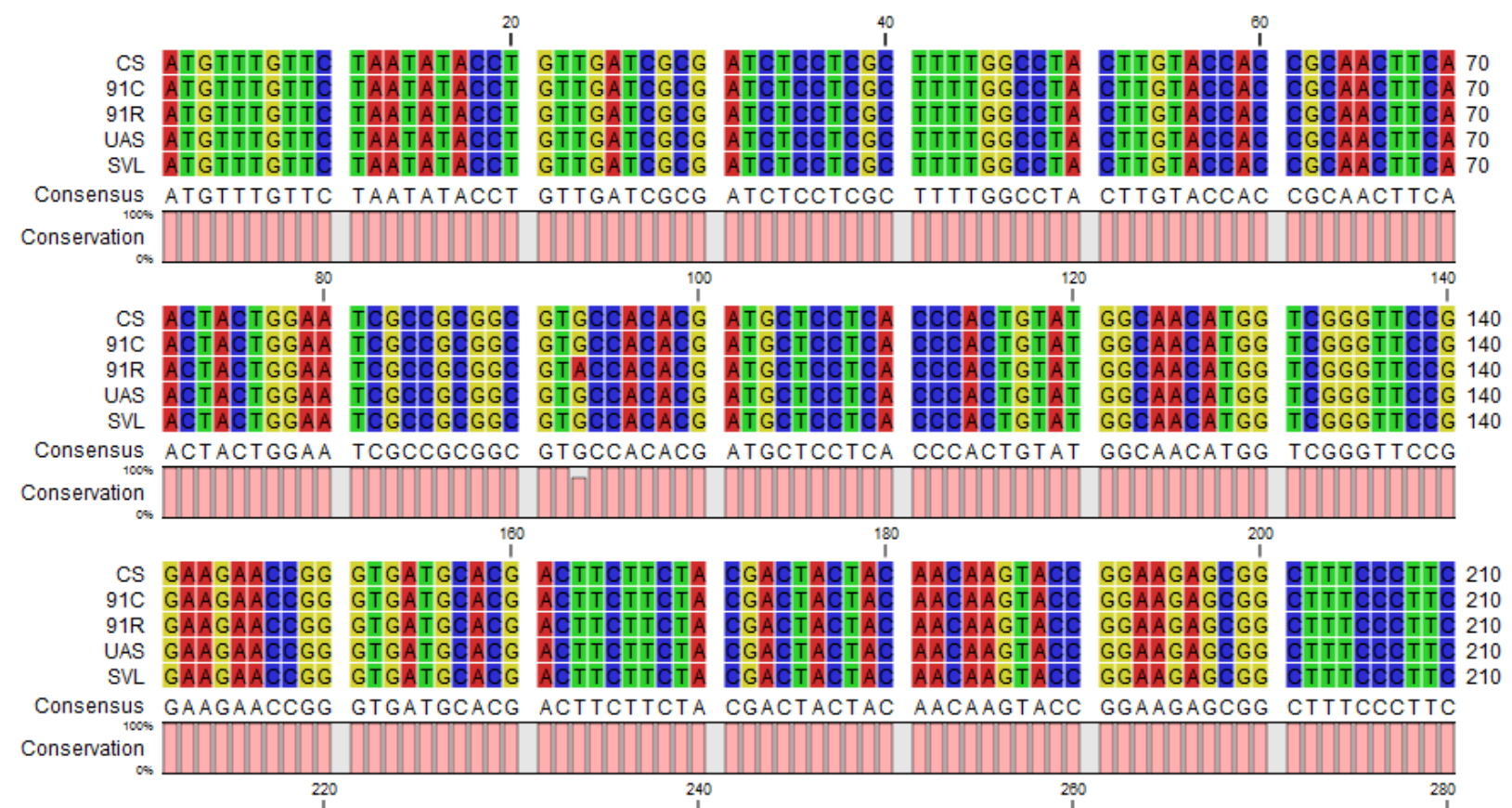

CS GTGGGCTTTI ACTTTCTGCA CAAGCCGGCC GCCTTCATCG TGGACACCCA GCTGGCCAAG AACATCCTGA 280 91C GTGGGCTTTT ACTTTCTGCA CAAGCCGGCC GCCTTCATCG TGGACACCCA GCTGGCCAAG AACATCCTGA 280 91R GTGGGCTTTT ACTTTCTGCA CAAGCCGGCC GCCTTCATCG TGGACACCCA GCTGGCCAAG AACATCCTGA 280 UAS GTGGGCTTTT ACTTTCTGCA CAAGCCGGCC GCCTTCATCG TGGACACCCA GCTGGCCAAG AACATCCTGA 280

SVL GTGGGCTTTT ACTTTCTGCA CAAGCCGGCD GCCTTCATCG TGGACACCCA GCTGGCCAAG AACATCCTGA 280 Consensus GTGGGCTTTT ACTTTCTGCA CAAGCCGGCC GCCTTCATCG TGGACACCCA GCTGGCCAAG AACATCCTGA Conservation

$$
300
$$
320 340

CS TCAAGGATTT CTCGAACTTT GCCGATCGTG GCCAGTTTCA CAACGGGCGC GACGACCCGC TCACGCAGCA 350 91C TCAAGGATTT CTCGAACTTT GCCGATCGTG GCCAGTTTCA CAACGGGCGC GACGACCCGC TCACGCAGCA 350 91R TCAAGGATTT CTCGAACTTT GCCGATCGTG GCCAGTTTCA CAACGGGCGC GACGACCCGC TCACGCAGCA 350 UAS TCAAGGATTT СTCGAACTTT GCCGATCGTG GCCAGTTTCA CAACGGGCGC GACGACCCGC TCACGCAGCA 350

SVL TCAAGGATTT СTCGAATTTT GCCGATCGTG GCCAGTTTCA CAACGGGCGD GACGACCCGD TCACGCAGCA 350 Consensus TCAAGGATTT CTCGAACTTT GCCGATCGTG GCCAGTTTCA CAACGGGCGC GACGACCCGC TCACGCAGCA

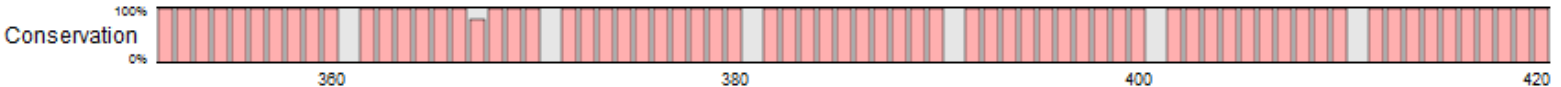

CS CCTGTTCAAC CTGGACGGAA AGAAGTGGAA GGACATGCGC CAGAGGCTGA CGCCGACGTT CACCTCGGGC 420 $91 \mathrm{C}$ СCTGTTCAAC СTGGACGGAA AGAAGTGGAA GGACATGCGE CAGAGGCTGA CGCCGACGTT CACCTCGGGC 420 91R CCTGTTCAAC CTGGACGGAA AGAAGTGGAA GGACATGCGC CAGAGGCTGA CGCCGACTTT CACCTCGGGC 420 UAS CCTGTTCAAC CTGGACGGAA AGAAGTGGAA GGACATGCGC CAGAGGCTGA CGCCGACGTT CACCTCGGGC 420

SVL CCTGTTCAAC CTTGACGGAA AGAAGTGGAA GGACATGCGC CAGAGGCTGA CGCCGACTTT CACCTCGGGD 420 Consensus CCTGTTCAAC CTGGACGgA AGAAGTGGA GGACATGCGC CAGAGGCTGA CGCCGACGTT CACCTCGGGC

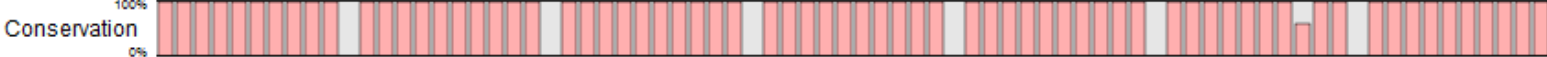
440 460

CS AAGATGAAGT TCATGTTCCC GACGGTGATC AAGGTGTCCG AGGAGTTCGT CAAGGTGATC ACGGAGCAGG 490 91C AAGATGAAGT TCATGTTCCC GACGGTGATC AAGGTGTCCG AGGAGTTCGT CAAGGTGATC ACGGAGCAGG 490 91R AAGATGAAGT TCATGTTCCD GACGGTGATC AAGGTGTCCG AGGAGTTCGT CAAGGTGATC ACGGAGCAGG 490 UAS AAGATGAAGT TCATGTTCCC GACGGTGATC AAGGTGTCCG AGGAGTTCGT CAAGGTGATC ACGGAGCAGG 490

SVL AAGATGAAGT TCATGTTCCC GACGGTGATC AAGGTGTCCG AGGAGTTCGT CAAGGTGATC ACGGAGCAGG 490 Consensus AAGATGAAGT TCATGTTCCC GACGGTGATC AAGGTGTCCG AGGAGTTCGT CAAGGTGATC ACGGAGCAGG Conservation

CS TGCCCGCCGC CCAGAACGGC GCTGTGCTCG AGATCAAGGA GCTGATGGCC AGGTTCACCA CCGATGTGAT 560 91C TGCCCGCCGC CCAGAACGGC GCTGTGCTCG AGATCAAGGA GCTGATGGCC AGGTTCACCA CDGATGTGAT 560 91R TGCCCGCCGC CCAGAACGGC GCTGTGCTCG AGATCAAGGA GCTGATGGCC AGGTTCACCA CCGATGTGAT 560 UAS TGCCCGCCGC CCAGAACGG GCTGTGCTCG AGATCAAGGA GCTGATGGCC AGGTTCACCA CDGATGTGAT 560

SVL TGCCCGCCGC CCAGAACGGC GCTGTGCTCG AGATCAAGGA GCTGATGGCD AGGTTCACCA CDGATGTGAT 560

Consensus TGCCCGCCGC CCAGAACGgC GCTGTGCTCG AGATCAAGgA GCTGATGGCC AGgTTCACCA CCGATGTGAT

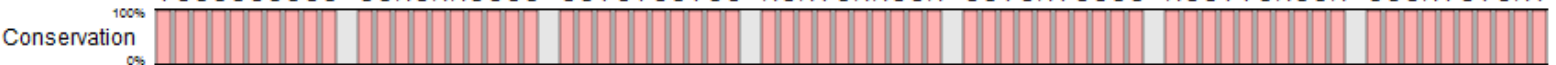




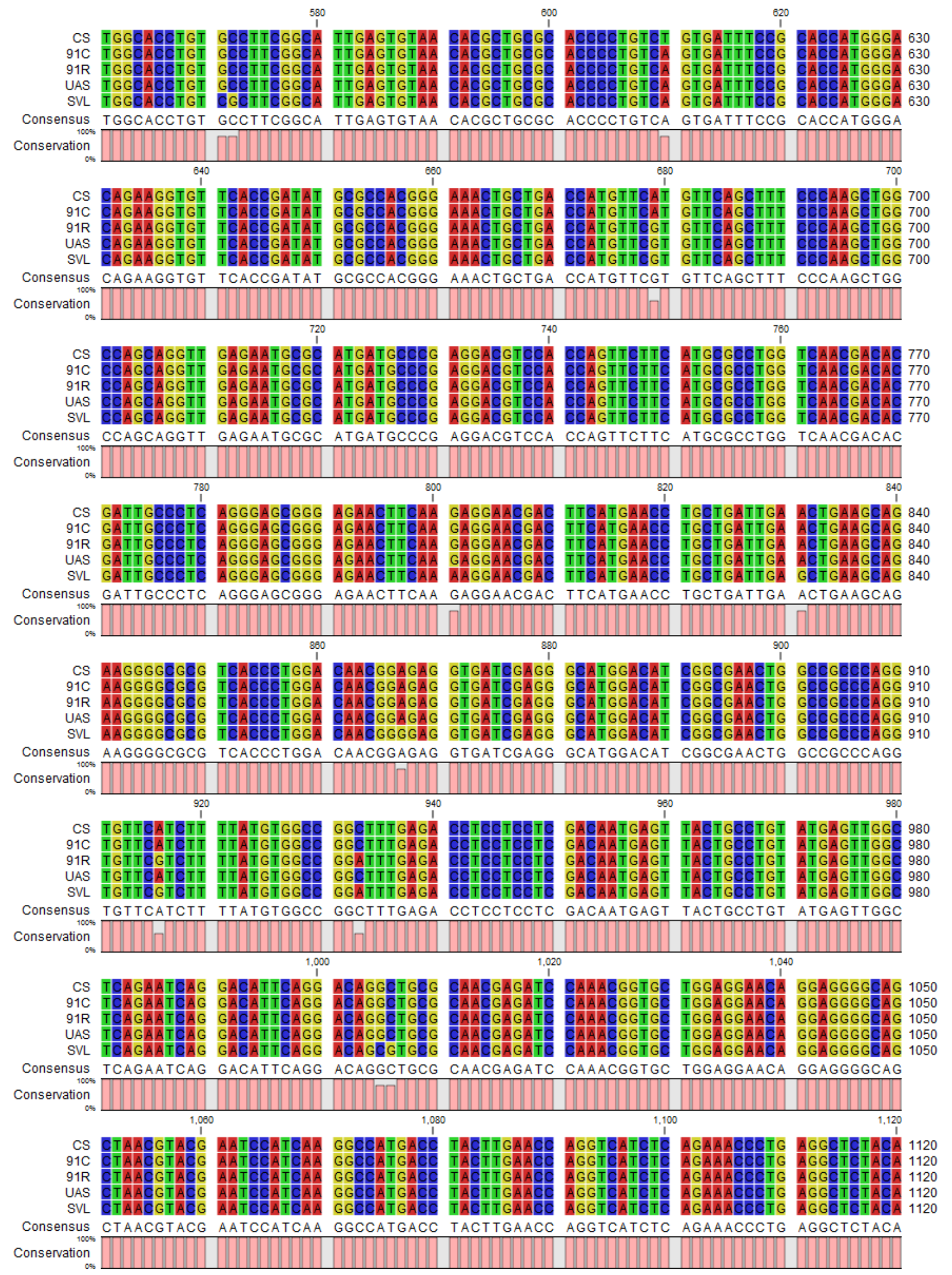




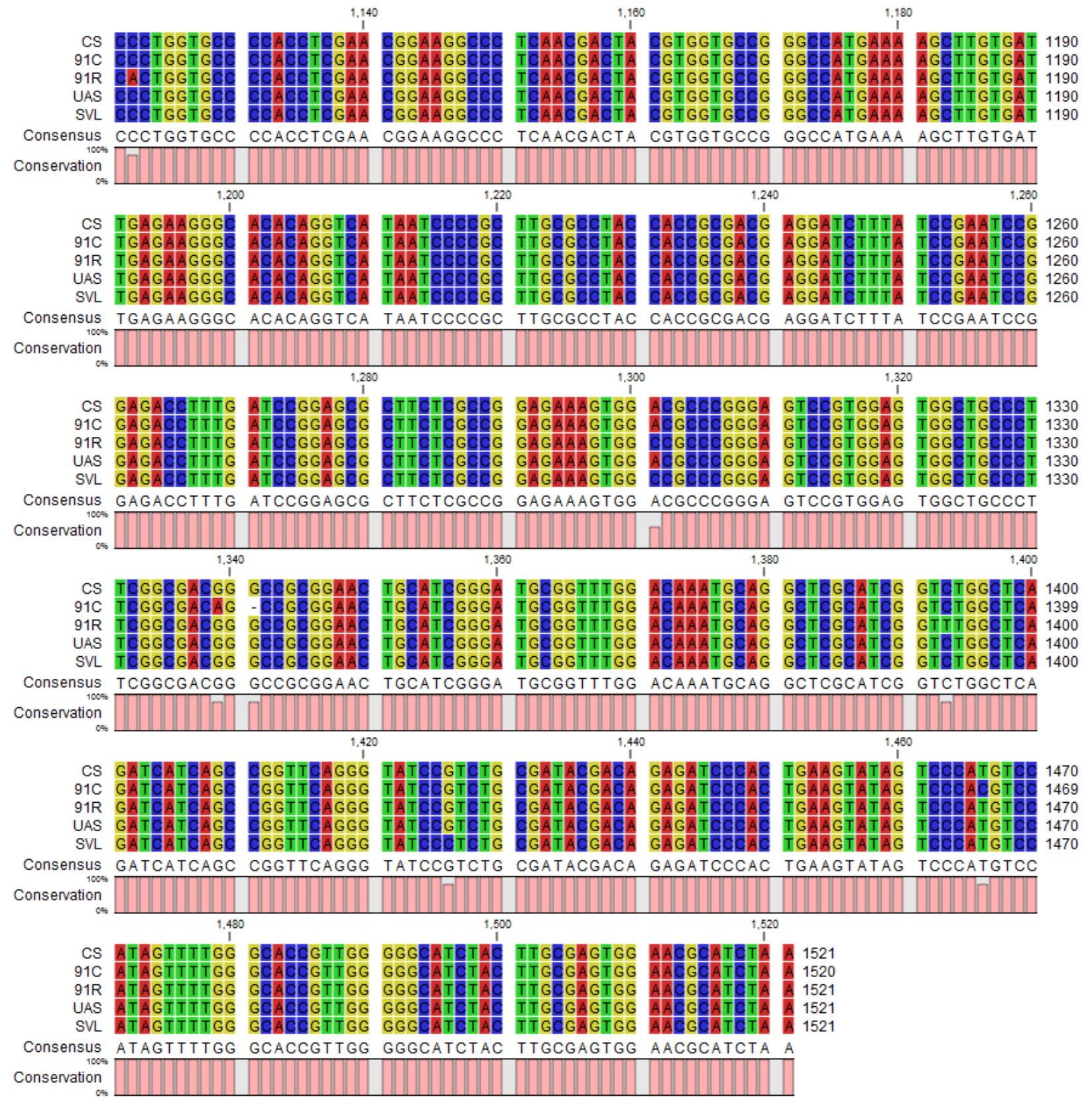

Figure 13. The CLC DNA sequence alignments between the $C S, 91-C, 91-R$, UAS-Cyp6a2, and Cyp6a2SVL strains showed 21 SNP's were present in the coding region of Cyp6a2. The overall consensus is signified by a pink bar underneath the sequences. The highly DDTresistant $91-R$ line and the Cyp6a2SVL line have mutations in common at nucleotide positions $408,916,933$, and 1301 . The $91-C$ strain contained a single basepair deletion at position nucleotide 1341. 


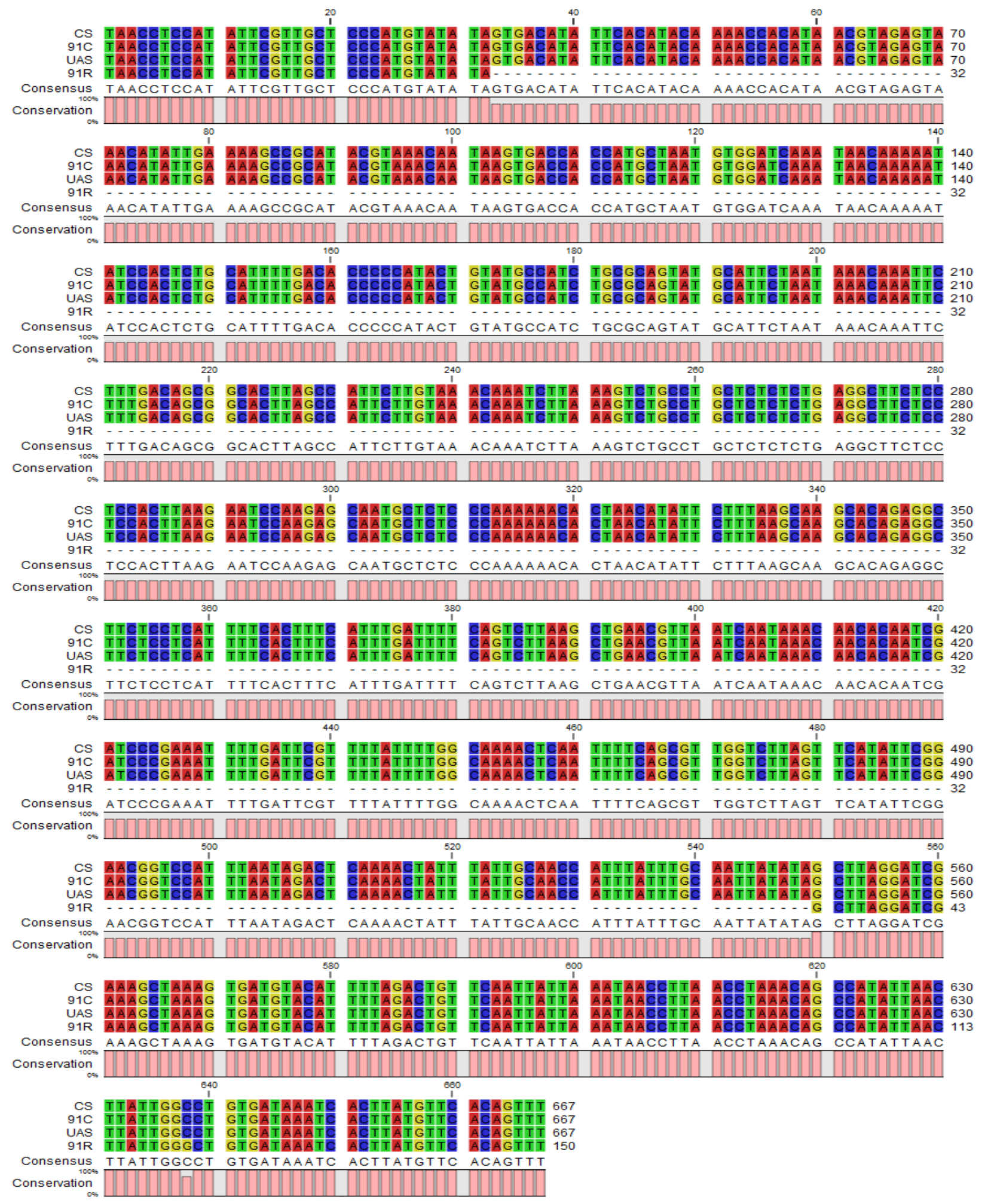

Figure 14. The CLC DNA sequence alignments between the $C S, 91-C$, UAS-Cyp6a2, and 91-R strains showed a $\sim 500$ bp insert in the 3 ' region of the gene that was not present in the DDTresistant $91-\mathrm{R}$ strain. The overall consensus is signified by a pink bar underneath the sequences, starting with TTA, the stop codon. 


\subsection{Protein sequence analysis}

While 21 SNP's were detected, only 9 of these point mutations led to amino acid changes (Table 5). The Cyp6a2SVL mutant, which is able to metabolize DDT, is named as such for the R335S, L336V, and V476L mutations [56]. These mutations were not found in the highly resistant $91-R$ line. However, there are several additional mutations of interest present (Fig. 15). At amino acid position 191, the Cyp6a2SVL had an A to R substitution. Amino acid positions 204, 227, 306, 336, and 476 all resulted in amino acid substitutions, however, the character of the resulting amino acid substitution was the same (i.e., polar to polar, non-polar to non-polar). At amino acid position 434, both the 91-R and Cyp6a2SVL had a D to A substitution, which may result in a significant change in the local protein structure and function. Similarly, at amino acid position 489 , there was a $\mathrm{M}$ to $\mathrm{T}$ mutation in the $91-C$ line. The $91-C$ sequence becomes degenerate from the rest at amino acid position 447 due to the base pair deletion, which led to a frame shift mutation.

\section{Table 5}

Sequence analysis of Cyp6a2 in DDT susceptible (CS, 91-C, UAS-Cyp6a2) and resistant (91-R, Cyp6a2SVL) strains led to the identification of several mutations in addition to SVL.

\begin{tabular}{cccccccccc}
\hline Strain & 191 & 204 & 227 & 306 & $\mathbf{3 3 5}^{*}$ & $\mathbf{3 3 6}^{*}$ & 434 & $\mathbf{4 7 6}^{*}$ & 489 \\
\hline$C S$ & $\mathrm{~A}$ & $\mathrm{C}$ & $\mathrm{M}$ & $\mathrm{I}$ & $\mathrm{R}$ & $\mathrm{L}$ & $\mathrm{D}$ & $\mathrm{V}$ & $\mathrm{M}$ \\
$91-C$ & $\mathrm{~A}$ & $\mathrm{~S}$ & $\mathrm{M}$ & $\mathrm{I}$ & $\mathrm{R}$ & $\mathrm{L}$ & $\mathrm{D}$ & $\mathrm{V}$ & $\mathrm{T}$ \\
$91-R$ & $\mathrm{~A}$ & $\mathrm{~S}$ & $\mathrm{~V}$ & $\mathrm{~V}$ & $\mathrm{R}$ & $\mathrm{L}$ & $\mathrm{A}$ & $\mathrm{V}$ & $\mathrm{M}$ \\
UAS-Cyp6a2 & $\mathrm{A}$ & $\mathrm{S}$ & $\mathrm{V}$ & $\mathrm{I}$ & $\mathrm{R}$ & $\mathrm{L}$ & $\mathrm{D}$ & $\mathrm{V}$ & $\mathrm{M}$ \\
Cyp6a2SVL & $\mathrm{R}$ & $\mathrm{S}$ & $\mathrm{V}$ & $\mathrm{V}$ & $\mathrm{S}^{*}$ & $\mathrm{~V}^{*}$ & $\mathrm{~A}$ & $\mathrm{~L}$ & $\mathrm{M}$ \\
\hline
\end{tabular}

Orange $=$ Non-polar, Blue $=$ Polar, Red $=$ Polar Charged $(+)$, Black $=$ Polar Charged $(-)$

* Indicates the amino acid positions of the Cyp6a2SVL mutations 


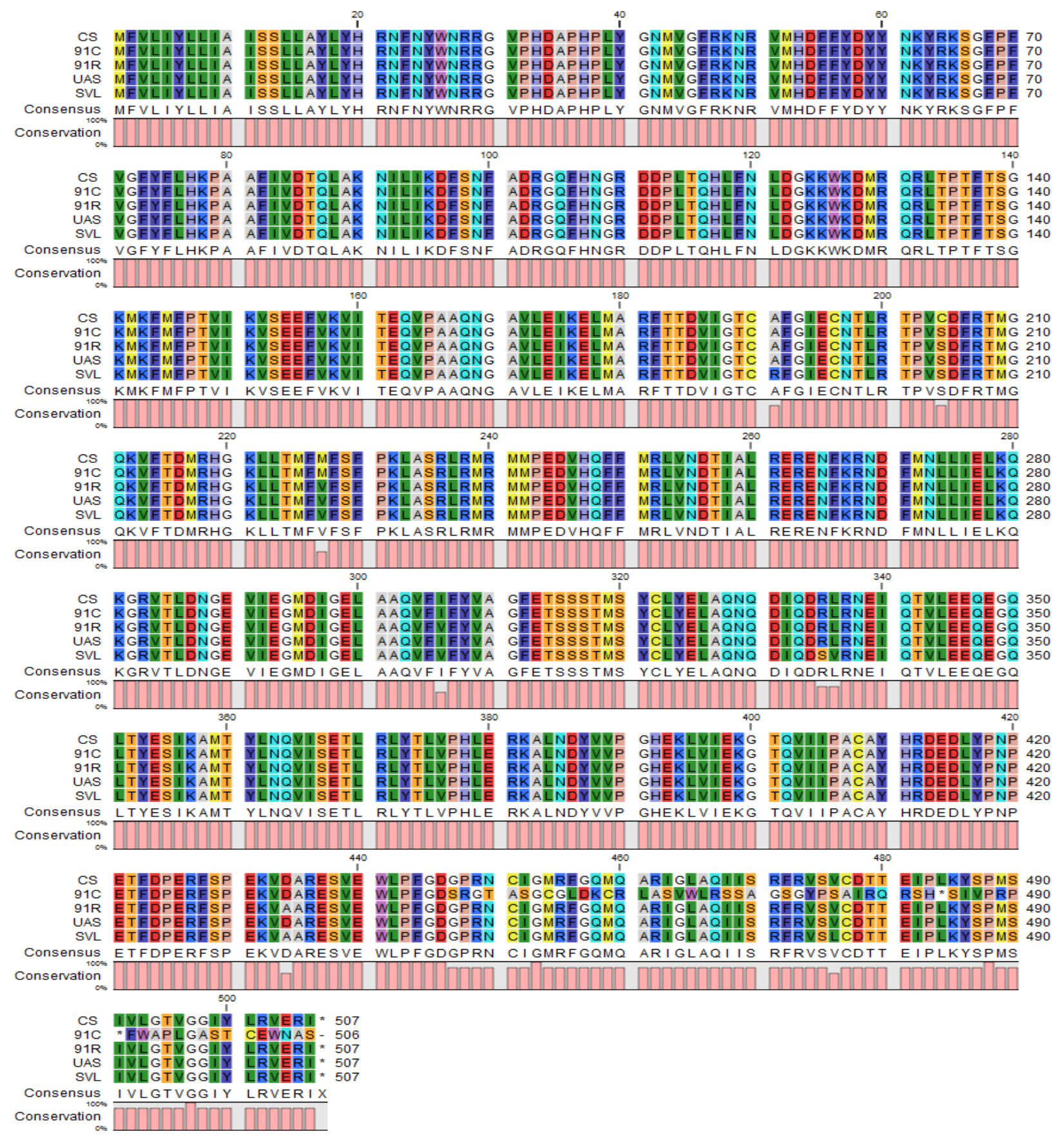

Figure 15. The CLC Protein sequence alignments between the $C S, 91-C, 91-R$, UAS-Cyp6a2, and Cyp6a2SVL strains showed 9 amino acid substitution in total. The overall consensus is signified by a pink bar underneath the sequences. The SVL amino acid substitutions (R335S / L336V / V476L) were only present in the Cyp6a2SVL line, however, the DDT-resistant 91-R and the Cyp6a2SVL strains shared two mutations in common (I306C /D434A). At this time it is unclear which of these mutations may enable Cyp6a2 to metabolize DDT. The 91-C sequence is degenerate from 447 onward due to a base pair deletion leading to a frame shift mutation. 


\subsection{Discussion}

In total, 21 SNP's were detected across the sequenced strains. Only 5 of these SNP's were found across multiple sequenced lines. These 21 SNP's led to a total of 9 amino acid substitutions. Of these 9 amino acid substitutions, 4 led to changes in the amino acid's character (i.e., polar to non-polar). The Cyp6a2SVL mutant, which can metabolize DDT, did not share the SVL mutations in common with any of the other sequenced lines, even the highly DDT-resistant 91-R. Interestingly, 2 novel mutations were found in common between the highly DDT-resistant 91-R line and the Cyp6a2SVL line. These 2 mutations resulted in amino acid substitutions at position I306V and position D434A. While the Cyp6a2SVL mutations were only found in the Cyp6a2SVL line, it is possible that the other mutations in common between the $91-R$ and Cyp6a2SVL may enable this particular Cyp6a2 variant to metabolize DDT directly. Further analysis is needed to determine the effect of the 3' UTR transposon insert as well as the effect of the amino acid substitutions. It is likely that the lack of the $3^{\prime}$ transposon in the $91-R$ line at least partially explains the higher transcript level found in the $91-R$. Further testing would need to be done to show prove this, however. 


\section{CHAPTER 5}

\section{SUMMARY AND FUTURE DIRECTIONS}

Of the 17 genes tested, 7 were found to have a significant effect on susceptibility to DDT as determined by UAS-RNAi (Fig. 7). Knockdown of Cyp4g1 was found to increase susceptibility to DDT by $25 \%$ and this gene is located on the X chromosome (chromosome 1); the only sex-linked resistance factor that has been determined to date. Lcp1, Cyp6g1, Cyp12d1, and Mdr50 are located on chromosome 2R and Mrpl is located on the left arm (2L) of chromosome 2. UAS-RNAi resulted in a $14 \%, 17 \%, 11 \%, 13 \%$ and $15 \%$ increase in susceptibility to DDT, respectively, making this chromosome prominent in DDT resistance. Mdr65 is located on chromosome $3 \mathrm{~L}$ and its knockdown by UAS-RNAi resulted in a $12 \%$ increase in susceptibility to DDT. While 10 of the genes tested by UAS-RNAi resulted in no increase in susceptibility to DDT, that does not mean that these genes play no role in DDT resistance. Instead, it may be an artifact of inefficient RNAi with the experimental conditions used (heat shock-inducible RNAi). To test this hypothesis, a constitutive Gal4 driver could be used in order to maximize chances of dsRNA induced RNAi. Additionally, constitutive over expression of all the genes tested should be tried, in order to further correlate the expression of these genes with DDT tolerance and resistance.

Multiple genes on all three chromosomes appear to be involved in DDT resistance in the highly resistant $91-R$ strain. These results are consistent with the original suggestion that DDT resistance in the $91-R$ strain is multifactorial and that factors on all three chromosomes are involved in DDT resistance [8-11]. Factors on chromosome 2 were suggested to play a major role, followed by factor(s) on chromosome 3, with the factor(s) on chromosome 1 showing a 
much smaller but statistically significant effect [19]. It was also suggested that the factors on chromosomes 2 and 3 interacted in a more than additive manner. Our results therefore suggest that the Cyp4g1 gene on chromosome 1 is a likely candidate as the resistance factor associated with this sex chromosome. Also Mdr65 on chromosome 3 is likely a resistance factor and may interact with either the $\mathrm{P} 450$ genes or the other $\mathrm{ABC}$ transporter genes on chromosome 2 in a synergistic fashion, resulting in extremely high levels of resistance. This conjecture, however, will need future experimental proof.

What is not yet known is how these resistance mechanisms interact with each other. Will it be in a less than additive, additive, or greater than additive manner? By injecting dsRNA into $91-R$ flies for each of the identified 7 genes individually, and then in combination, we will be able to determine how these multiple resistance mechanisms, found across all three chromosomes, interact.

While Cyp6a2 RNAi in the UAS-RNAi cross did not result in an increased susceptibility to DDT, the possibility still remains that Cyp6a2 in the $91-R$ line may be able to process DDT. As Cyp6a2 is one of the highest over transcribed (over 30-fold) P450s in the DDT-resistant $91-R$ strain [38], the energetic cost of such expression should be balanced with a gain in fitness upon exposure to DDT. One possible explanation for this over transcription may be due to the changes in the 3' UTR of Cyp6a2 in the 91-R line. While all DDT-susceptible (CS, 91-C, UAS-Cyp6a2) lines had a $\sim 500$ bp insertion in the 3' region of the Cyp6a2 gene, the DDT-resistant $91-R$ did not possess this insertion. Future studies should be done to evaluate the effect of this insertion, or lack thereof, on gene expression levels. 
The reported Cyp6a2SVL mutant was able to metabolize DDT when expressed in E. coli [56]. While the Cyp6a2SVL amino acid substitutions were not found to be present in the 91-R, there were still 2 amino acid substitutions shared between the DDT-resistant $91-R$ and the Cyp6a2SVL strains. The I306V and D434A mutations were both found in the $91-R$ and Cyp6a2SVL strains. These mutations, however, were not present in the susceptible strains (CS, 91-C, UAS-Cyp6a2). The I306V amino acid substitution, while found in both the $91-R$ and Cyp6a2SVL strains, results in a non-polar to non-polar amino acid substitution, which may not drastically change the structure of the resulting protein. Of more interest, the D434A mutation results in polar charged (aspartic acid) to non-polar hydrophobic (alanine) substitution. It is possible that this change may change the local structure of Cyp6a2 allowing it to more efficiently bind DDT for phase I xenobiotic metabolism. Further, site directed mutagenesis studies should be run to evaluate the effect of these mutations in the ability of Cyp6a2 to metabolize DDT. 


\section{REFERENCES}

[1] World Health Organization. Indoor residual spraying: use of indoor residual spraying for scaling up global malaria control and elimination, Geneva, Switzerland. (WHO/HTM/MAL/ 2006.1112).

[2] Hemingway, J., \& Ranson, H. Insecticide Resistance in Insect Vectors of Human Disease. Annual Review of Entomology, (2000) 45, 1

[3] Prato, Mauro, Manuela Polimeni, Giuliana Giribaldi. DDT as Anti-Malaria Tool: The Bull in the China Shop or the Elephant in the Room?, Insecticides - Development of Safer and More Effective Technologies, Prof. Stanislav Trdan (2013) ISBN: 978-953-51-0958-7, InTech, DOI: $10.5772 / 53241$.

[4] R.L. Metcalf. Insect resistance to insecticides. Pestic. Sci. 26.4 (1989) 333-358.

[5] J.D. Edman. Emerging vectorborne diseases and their control. In: J.M. Clark and H. Okhawa (Eds.). New Discoveries in Agrochemicals. ACS Symposium Series 892. ACS Books. Washington, D.C. (2004) pp. 314-325.

[6] J. Hemingway. Vector biology diagnostics and public health pesticide development through the product development partnership route. In: J.M. Clark, J.R. Bloomquist and H. Kawada (Eds.). Advances in Vector Human Vector Control. ACS Symposium Series 1014. ACS Books, Washington D.C. (2009) pp.1-12.

[7] T.G. Wilson. Drosophila melanogaster (Diptera: Drosophilidae): a model insect for insecticide resistance studies. J. Econ. Entomol. 81 (1988) 22-27.

[8] J.F. Crow. Analysis of a DDT-resistant strain of Drosophila. J. Econ. Entomol.47 (1954) 393-398.

[9] D.J. Merrell, J.C. Underhill. Selection of DDT resistance in inbred, laboratory and wild stocks of Drosophila melanogaster. J. Econ. Entomol. 49 (1956) 300-306.

[10] D. Dapkus, D.J. Merrell. Chromosomal analysis of DDT-resistance in a long-term selected population of Drosophila melanogaster. Genetics, 87 (1977) 685-697.

[11] D. Dapkus. Genetic localization of DDT resistance in Drosophila melanogaster (Diptera: Drosophilidae). J. Econ. Entomol. 85 (1992) 340-347.

[12] Bolshakov, V. N., Topalis, P., Blass, C., Kokoza, E., della, T. A., Kafatos, F. C., \& Louis, C. A comparative genomic analysis of two distant diptera, the fruit fly, Drosophila melanogaster, and the malaria mosquito, Anopheles gambiae. Genome Research, 12, 1, (2002) 57-66.

[13] Joseph P. Strycharz, Alice Lao, Hongmei Li, Xinghui Qiu, Si Hyeock Lee, Weilin Sun, Kyong Sup Yoon, Jeffery J. Doherty, Barry R. Pittendrigh, J. Marshall Clark. Resistance in the highly DDT-resistant $91-R$ strain of Drosophila melanogaster involves decreased penetration, increased metabolism, and direct excretion. Pestic. Biochem. and Physiol. 107 (2013) 207-217. 
[14] J.H.F. Pedra, L.M. McIntyre, M.E. Scharf, B.R. Pittendrigh. (2004). Genome-wide transcription profile of field- and laboratory-selected dichlorodiphenyltrichloroethane (DDT)resistant Drosophila. PNAS. 101 (2004) 7034-7039.

[15] X. Qiu, W. Sun, C.M. McDonnell, H. Li-Byarlay, L.D. Steele, J. Wu, J. Xie, W.M. Muir, B.R. Pittendrigh. Genome-wide analysis of genes associated with moderate and high DDT resistance in Drosophila melanogaster. Pest Manag. Sci. (2013) DOI 10.1002/ps.3454.

[16] Pedra, J. H., Festucci-Buselli, R. A., Sun, W., Muir, W. M., Scharf, M. E., \& Pittendrigh, B. R. Profiling of abundant proteins associated with dichlorodiphenyltrichloroethane resistance in Drosophila melanogaster. Proteomics, 5(1), (2005) 258-269.

[17] B.R. Pittendrigh, R. Reenan, R.H. ffrench-Constant, B. Ganetzky. Point mutation in the Drosophila sodium channel gene para associated with resistance to DDT and pyrethroid insecticides. Mol. Gen. Genet. 256 (1997) 602-610.

[18] R. Feyereisen. Insect P450 Enzymes. Annu. Rev. Entomol. 44 (1999) 507-533.

[19] R. Feyereisen. Insect Cytochrome P450. In: L.I. Gilbert, K. Iatrou, S.S. Gill (Eds.). Comprehensive Molecular Insect Science. Elsevier, Amsterdam. (2005) pp. 1-77.

[20] G. Dietzl, D. Chen, F. Schnorrer, K.C. Su, Y. Barinova, M. Fellner, B. Gasser, B.J. Dickson, A genome-wide transgenic RNAi library for conditional gene inactivation in Drosophila. Nature. 448 (2007) 151-156.

[21] D.A. Elliott, A.H. Brand. The GAL4 System. Methods Mol. Biol. 420 (2011) 79-95.

[22] Elliott DA, Brand AH. The GAL4 system : a versatile system for the expression of genes. Methods Mol Biol. (2011) 420:79-95.

[23] Haley B, Tang G, Zamore. In vitro analysis of RNA interference in Drosophila melanogaster. Methods 30: (2003) 330-336.

[24] R.M. Sawicki, A.W. Farnham. (1968). Genetics of resistance to insecticides of the ska strain of Musca domestica. Entomol. Experim. Et. Appl. 11 (1968) 133-142.

[25] R.D. O’Brien, I. Yamamoto. Biochemical toxicology of insecticides. New York, NY, Academic Press, 93 (1970) 105-111.

[26] Gibbs, A. G. Water-proofing properties of cuticular lipids. American Zoologist, 38, 3, (1998) 471-482.

[27] Nation, J. L. Insect physiology and biochemistry. (2002) Boca Raton: CRC Press.

[28] Y. Qiu, C. Tittiger, C. Wicker-Thomson, G. Le Goff, S. Young, E. Wajnberg, T. Fricaux, N. Taquet, G.J. Blomquist, R. Feyereisen. An insect-specific P450 oxidative decarbonylase for cuticular hydrocarbon biosynthesis. PNAS, 109 (2012) 14858-14863. 
[29] F.W. Plapp, R.F. Hoyer. Insecticide resistance in the house fly: decreased rate of absorption as the mechanism of action of a gene that acts as an intensifier of resistance. J. Econ. Entomol. 61 (1968) 1298-1303.

[30] C. Pan, Y. Zhou, J. Mo. The clone of laccase gene and its potential function in cuticular penetration resistance of Culex pipiens pallens to fenvalerate. Pestic. Biochem. Physiol. 93 (2009) 105-111.

[31] M. Snyder, J. Hirsh N. Davidson. The cuticular genes of Drosophila: A developmentally regulated gene cluster. Cell, 25 (1981) 165-177.

[32] Richards, A. G., \& Cutkomp, L. K. Correlation between the possession of a chitinous cuticle and sensitivity to DDT. The Biological Bulletin, 90(2), (1946) 97-108.

[33] Locke, Michael. (1961). Pore Canals and Related Structures in the Insect Cuticle. Rockefeller University Press.

[34] Kitamura, S., Shimizu, Y., Shiraga, Y., Yoshida, M., Sugihara, K., \& Ohta, S. Reductive Metabolism ofp, p'-DDT ando, p'-DDT by Rat Liver Cytochrome P450. Drug metabolism and disposition, 30(2), (2002) 113-118.

[35] LANGEN, H., EPPRECHT, T., LINDEN, M., HEHLGANS, T., GUTTE, B., \& BUSER, H. R.. Rapid partial degradation of DDT by a cytochrome P-450 model system. European Journal of Biochemistry, 182(3), (1989) 727-735.

[36] P. Daborn, S. Boundy, J. Yen, B. Pittendrigh, R.H. ffrench-Constant. DDT resistance in Drosophila correlates with Cyp6gl over-expression and confers cross-resistance to the neonicotinoid imidacloprid. Mol. Genet. Genomics, 266 (2001) 556-563.

[37] R.A. Festucci-Buselli, A.S. Carvalho-Dias, M. de Oliveira-Andrade, C. Caixeta-Nunes, H.M. Li, J.J. Stuart, W. Muir, M.E. Scharf, B.R. Pittendrigh. Expression of Cyp6gl and Cyp12d1 in DDT resistant and susceptible strains of Drosophila melanogaster. Insect Mol. Biol. 14 (2005) 69-77.

[38] Maitra, Sushmita, et al. Factors on the third chromosome affect the level of Cyp6a2 and Cyp6a8 expression in Drosophila melanogaster. Gene 248.1 (2000) 147-156.

[39] Wan, Hua, et al. Nrf2/Maf-binding-site-containing functional Cyp6a2 allele is associated with DDT resistance in Drosophila melanogaster. Pest management science 70.7 (2014) 1048-1058.

[40] G. Le Goff, S. Boundy, P.J. Daborn, J.L. Yen, L. Sofer, R. Lind, C. Sabourault, L. MadiRavazzi, R.H. ffrench-Constant. Microarray analysis of cytochrome P450 mediated insecticide resistance in Drosophila. Insect Biochem. Mol. Biol. 33 (2003) 701-708. 
[41] Joußen, N., Heckel, D. G., Haas, M., Schuphan, I., \& Schmidt, B. Metabolism of imidacloprid and DDT by P450 CYP6G1 expressed in cell cultures of Nicotiana tabacum suggests detoxification of these insecticides in Cyp6g1-overexpressing strains of Drosophila melanogaster, leading to resistance. Pest management science, 64(1), (2008) 65-73.

[42] P.J. Daborn, J.L. Yen, M.R. Bogwitz, G. Le Goff, E. Feil, S. Jeffers, N. Tijet, T. Perry, D. Heckel, P. Batterham, R. Feyereisen, T.G. Wilson, R.H. ffrench Constant. A single P450 allele associated with insecticide resistance in Drosophila. Science, 297 (2002) 2253-2256.

[43] P.J. Daborn, C. Lumb, A. Boey, W. Wong, R.H. ffrench-Constant, P. Batterham. Evaluating the insecticide resistance potential of eight Drosophila melanogaster cytochrome P450 genes by transgenic over-expression. Insect Biochem. Mol. Biol. 37 (2007) 512-519.

[44] Morra, R., Kuruganti, S., Lam, V., Lucchesi, J. C., \& Ganguly, R. Functional analysis of the cis-acting elements responsible for the induction of the Cyp6a8 and Cyp6g1 genes of Drosophila melanogaster by DDT, phenobarbital and caffeine. Insect molecular biology, 19(1), (2010) 121-130.

[45] F. Catania, M.O. Kauer, P.J. Daborn, J.L. Yen, R.H. ffrench-Constant, C. Schlotterer. World-wide survey of an Accord insertion and its association with DDT resistance in Drosophila melanogaster. Mol. Gene. Genom. 266 (2004) 2491-2504.

[46] J.M. Schmidt, R.T. Good, B. Appleton, J. Sherrard, G.C. Raymant, M.R. Bogwitz, J. Martin, P.J. Daborn, M.E. Goddard, P. Batterham. Copy number, variation and transposable elements feature in recent ongoing adaptation at the Cyp6gl locus. Plos genetics 6 (2010) e1000998, pp.1-11.

[47] Chung, H., Bogwitz, M. R., McCart, C., Andrianopoulos, A., Batterham, P., \& Daborn, P. J. Cis-regulatory elements in the Accord retrotransposon result in tissue-specific expression of the Drosophila melanogaster insecticide resistance gene Cyp6g1. Genetics, 175(3), (2007) 1071-1077.

[48] S. Kuruganti, V. Lam, X. Zhou, G. Bennett, B.R. Pittendrigh, R. Ganguly. High expression of Cyp6g1, a cytochrome P450 gene, does not necessarily confer DDT resistance in Drosophila melanogaster. Gene, 388 (2007) 43-53.

[49] McCart, C. Dissecting the insecticide-resistance-associated cytochrome P450 gene Cyp6g1. Pest management science, 64(6), (2008) 639-645.

[50] Brandt, A., Scharf, M., Pedra, J. H. F., Holmes, G., Dean, A., Kreitman, M., \& Pittendrigh, B. R. Differential expression and induction of two Drosophila cytochrome P450 genes near the Rst (2) DDT locus. Insect molecular biology, 11(4), (2002) 337-341.

[51] Maitra, S., Price, C., \& Ganguly, R. Cyp6a8 of Drosophila melanogaster: gene structure, and sequence and functional analysis of the upstream DNA. Insect biochemistry and molecular biology, 32(8), (2002) 859-870. 
[52] B.C. Dunkov, V.M. Guzov, G. Mocelin, F. Shotkoski, A. Brun, M. Amichot, R.H. ffrenchConstant, R. Feyereisen. The Drosophila cytochrome P450 gene Cyp6a2: structure, localization, heterologous expression, and induction by phenobarbital. DNA Cell Biol. 16 (1997) 1345-1356.

[53] S. Maitra, S. Dombrowski, L. Waters, R. Ganguly. Three second chromosome-linked clustered Cyp6 genes show differential constitutive and barbital-induced expression in DDTresistant and susceptible strains of Drosophila melanogaster, Gene 189 (1996) 165-171.

[54] A. Brun, A. Cuany, T. LeMouel, J.B. Berge, M. Amichot. Inducibility of the Drosophila melanogaster cytochrome P450 gene, CYP6A2, by phenobarbital in insecticide susceptible or resistant strains. Insect Biochem. Mol. Biol., 26 (1996) 697-703.

[55] Waters, L. C., Zelhof, A. C., Shaw, B. J., \& Ch'ang, L. Y. Possible involvement of the long terminal repeat of transposable element 17.6 in regulating expression of an insecticide resistance-associated P450 gene in Drosophila. Proceedings of the National Academy of Sciences, 89(11), (1992) 4855-4859.

[56] Amichot, Marcel, et al. Point mutations associated with insecticide resistance in the Drosophila cytochrome P450 Cyp6a2 enable DDT metabolism. European Journal of Biochemistry 271.7 (2004) 1250-1257.

[57] D.S. Buss, A. Callaghan. Interaction of pesticides with p-glycoprotein and other ABC proteins: A survey of the possible importance to insecticide, herbicide and fungicide resistance. Pestic. Biochem. Physiol. 90 (2008) 141-153.

[58] L. Podsiadlowski, V. Matha, A. Vilcinskas. (1998). Detection of a p-glycoprotein related pump in Chironomus larvae and its inhibition by verapamil and cyclosporine A. Comp. Biochem. Physiol. 121 (1998) 443-450.

[59] D.S. Buss, A.R. McCaffery, A. Callaghan. Evidence for p-glycoprotein modification of insecticide toxicity in mosquitoes of the Culex pipiens complex. Med. Vet. Entomol. 16 (2002) 218-222.

[60] R. Srinivas, S.S. Udikeri, S.K. Jayalakshmi, K. Sreeramulu, (2004). Identification of factors responsible for insecticide resistance in Helicoverpa armigera. Comp. Biochem. Physiol. Pt. C, 137 (2004) 261-269.

[61] D.J. Hawthorne and G.P. Dively. Killing them with kindness? In-hive medications may inhibit xenobiotic efflux transporters and endanger honey bees. PLoS ONE, 6 (2011) 10.1371/ journal. Pone 0026796.

[62] J.P. Strycharz, S.H. Lee, W. Sun, B.R. Pittendrigh, J.M. Clark. RNAi knockdown of ABC transporters causes decreased tolerance in the highly DDT-resistant $91-R$ strain of Drosophila melanogaster. "Picogram: Abstract Book", vol. 78, number 182, ACS/AGRO (2010) 161 pages, Page 116. 
[63] Vache, C., Camares, O., DeGraeve, F., Dastugue, B., Meiniel, A., Vaury, C., Pellier, S., Leoz-Garziandia, E., Bamdad, M. Drosophila melanogaster p-glycoprotein: a membrane detoxification system towards polycyclic aromatic hydrocarbon pollutants. Environ. Toxicol. Chem. 25 (2006) 572-580.

[64] Gerrard, B., Stewart, C., Dean, M. Analysis of Mdr50: A Drosophila p-glycoprotein / multidrug resistance gene homolog. Genomics. 17 (1993) 83-88.

[65] Wu, C.-T., Budding, M., Griffin, M.S., Croop, J.M. Isolation and characterization of Drosophila multidrug resistance gene homologs. Mol. Cell Biol. 11 (1991) 3940-3948.

[66] C.L. Lanning, R.L. Fine, J.L. Corcoran, H.M. Ayad, R.L. Rose, M.B. Abou-Donia. Tobacco budworm p-glycoprotein: biochemical characterization and its involvement in pesticide resistance. Biochem. Biophys. Acta. 1291 (1996) 155-162.

[67] Vache, C., Camares, O., Dastugue, B., Creveaux, I., Vaury, C., \& Bamdad, M. A potential genomic biomarker for the detection of polycyclic aromatic hydrocarbon pollutants: multidrug resistance gene 49 in Drosophila melanogaster. Environmental Toxicology and Chemistry, 26(7), (2007) 1418-1424.

[68] Tapadia, M. G., \& Lakhotia, S. C.Expression of mdr49 and mdr65 multidrug resistance genes in larval tissues of Drosophila melanogaster under normal and stress conditions. Cell stress \& chaperones, 10(1), (2005) 7.

[69] A. Shabbir, S. DiStasio, J. Zhao, C.P. Cardozo, M.S. Wolff, A.J. Caplan. Differential effects of the organochlorine pesticide DDT and its metabolite p,p'-DDE on p-glycoprotein activity and expression. Toxicol. Appl. Pharmacol. 203 (2005) 91-98.

[70] Tarnay, J.N., Szeri, F., Ilias, A., Annilo, T., Sung, C., LeSaux, O., Varadi, A., Dean, M., Boyd, C.D., Robinow, S. The dMRP/CG6214 gene of Drosophila is evolutionarily and functionally related to the human multidrug resistance associated protein family. Insect Mol. Biol. 13 (2004) 539-548.

[71] Raymond, M., Heckel, D.G., Scott, J.G. Interactions between pesticide genes: Model and experiment. Genetics., 123, (1989) 543-551.

[72] K.J. Livak, T.D. Schmittgen. Analysis of relative gene expression data using real-time quantitative PCR and the 2-??Ct Method. Methods 25 (2001) 402-408.

[73] Goto, A., Blandin, S., Royet, J., Reichhart, J. M., \& Levashina, E. A. Silencing of Toll pathway components by direct injection of double-stranded RNA into Drosophila adult flies. Nucleic acids research, 31(22), (2003) 6619-6623.

[74] Dzitoyeva, S., Dimitrijevic, N., \& Manev, H. Intra-abdominal injection of double-stranded RNA into anesthetized adult Drosophila triggers RNA interference in the central nervous system. Molecular psychiatry, 6(6), (2001) 665-670. 